\title{
Líquenes Parmelioides (Parmeliaceae, Ascomycota) del Parque Nacional Copo (Provincia de Santiago del Estero, Argentina)
}

\author{
MÓNICA T. ADLER ${ }^{1}$
}

\begin{abstract}
Summary: Parmelioid lichens (Parmeliaceae, Ascomycota) of National Park Copo (Santiago del Estero Province, Argentina). Twenty three species of foliose Parmeliaceae were collected and identified in Copo National Park and surroundings, on trunks and branches in primary and secondary forests of Aspidosperma quebracho-blanco and Schinopsis lorentzii, two tree species known as quebracho blanco and quebracho colorado respectively. The species are briefly described, including particular comments and the geographic distribution of each one of them. Hypotrachyna aspera C. H. Ribeiro \& Marcelli is reported for the first time for Argentina. Twelve species are also reported for the first time for Santiago del Estero Province. A key to differentiate the described species is proposed.
\end{abstract}

Key words: National Park Copo, Argentine lichens, Parmeliaceae, Hypotrachyna aspera.

\begin{abstract}
Resumen: Veintitrés especies de Parmeliaceae foliosas fueron coleccionadas en el Parque Nacional Copo y alrededores, sobre troncos y ramas en bosques vírgenes y secundarios de Aspidosperma quebracho-blanco y Schinopsis lorentzii, dos especies arbóreas conocidas como quebracho blanco y quebracho colorado respectivamente. Las especies se describen brevemente, incluyendo comentarios particulares y la distribución geográfica de cada una de ellas. Hypotrachyna aspera C. H. Ribeiro \& Marcelli se menciona por primera vez para la Argentina. Doce especies se citan también por primera vez para la Provincia de Santiago del Estero. Se propone una clave para diferenciar las especies descriptas.
\end{abstract}

Palabras clave: Parque Nacional Copo, líquenes argentinos, Parmeliaceae, Hypotrachyna aspera.

\section{INTRODUCCIÓN}

En este trabajo se denomina "líquenes parmelioides" al grupo de géneros así definidos por Crespo et al. (2010) que reúnen las siguientes características: talos mayormente foliosos y dorsiventrales, usualmente con ricinas en la superficie inferior, apotecios y picnidios en la superficie superior de la lámina, ascos del tipo Lecanora y ascosporas simples, hialinas, elipsoides. Otros géneros (p. ej. Usnea), incluidos dentro de Parmeliaceae por Tehler \& Wedin (2008) son denominados no parmelioides por Crespo et al. (2010). Según estos autores los líquenes parmelioides representan aproximadamente el 75\% de las especies descriptas dentro de la familia.

\footnotetext{
${ }^{1}$ PROPLAME-PRHIDEB (CONICET-UBA). Departamento de Biodiversidad y Biología Experimental. Facultad de Ciencias Exactas y Naturales. Universidad de Buenos Aires. Ciudad Universitaria, 1428 Buenos Aires. Argentina; e-mail: adler@bg.fcen.uba.ar
}

Existen estudios previos sobre líquenes del Noroeste argentino (NOA) y varias otras regiones de nuestro país, pero el conocimiento taxonómico de la micobiota liquenizada argentina es aún escasa (Calvelo \& Liberatore, 2002). Uno de los grupos con estudios actualizados en la Argentina lo constituyen las Parmeliaceae parmelioides, para las que se han publicado especies nuevas (e.g., Ferraro, 1979; Adler, 1987; Adler \& Elix, 1987; Elix \& Adler, 1987; Adler, 1989; Ferraro \& Elix, 1993; Calvelo \& Adler, 1999; Estrabou \& Adler, 2000; Ferraro \& Elix, 2000), así como estudios sobre el género Punctelia (Ferraro, 1986; Adler, 1996; Adler \& Ahti, 1996; Adler, 1997; Thell et al. 2005) y ampliación de distribuciones conocidas de varias especies (p. ej. Estrabou \& Adler, 1999; Calvelo \& Adler, 1999; Popoff \& Ferraro, 2002; Adler \& Calvelo, 2002, 2007, 2010; Michlig \& Ferraro, 2010; Michlig \& Ferraro, 2012a; Michlig \& Ferraro, 2012b).

Los principales objetivos de este trabajo fueron la identificación y descripción de las especies de 
Parmeliaceae parmelioides del Parque Nacional Copo (Santiago del Estero, NOA) y su diferenciación mediante una clave dicotómica, como aportes al conocimiento de la micobiota liquenizada de dicho Parque Nacional y al monitoreo de su conservación en el tiempo.

\section{Materiales y Métodos}

Área de colección. Este trabajo se basó en el estudio de material coleccionado a lo largo del año 1985, en el Parque Nacional Copo, situado en el extremo noreste de la Provincia de Santiago del Estero, al norte de la Argentina, creado como Parque Nacional en el año 2000 (Ley 25.366), con el fin de proteger una importante región del "Chaco Seco Santiagueño". La misma ya era un área protegida desde1968, conocida con el nombre de Reserva Natural Copo hasta 1993 en que pasó a ser Parque Provincial. Se encuentra situada en una zona boscosa del Chaco semiárido, incluido en el distrito Occidental de la Provincia Biogeográfica Chaqueña (Cabrera \& Willink, 1980).

Los bosques vírgenes en este gran bosque xeromórfico están dominados por Aspidosperma quebracho-blanco (quebracho blanco) y Schinopsis lorentzii (quebracho colorado santiagueño), acompañados por Prosopis nigra (algarrobo negro), Ziziphus mistol (mistol) y los arbustos Capparis retusa, Acacia praecox y Celtis pallida (Protomastro, 1988; Prado 1993, López de Casenave et al., 1995). Las localidades más cercanas al área de muestreo (ubicada en el extremo sudoeste del Parque) son Pampa de los Guanacos $\left(26^{\circ} 14^{\prime} 00^{\prime \prime} \mathrm{S}\right.$, $61^{\circ} 51^{\prime} 01^{\prime \prime} \mathrm{O}$, aproximadamente a $15 \mathrm{~km}$ al sur de la entrada del Parque) y Los Pirpintos (26 $07^{\prime} 56^{\prime \prime} \mathrm{S}$, $62^{\circ} 03^{\prime} 49^{\prime \prime} \mathrm{O}$ ), ambas sobre la ruta nacional 16 en el Dpto. Copo. En el área de estudio se encuentran también representados bosques secundarios, explotados en la década de 1940, además de los bosques vírgenes que ocupan la mayor parte del Parque (Protomastro, 1988).

Colecciones de líquenes. Los especímenes examinados fueron coleccionados principalmente en áreas con bosque virgen dentro del Parque (abreviado Copo en Material examinado) y en Los Pirpintos por Jorge J. Protomastro (JJP), quien obtuvo las muestras principalmente durante 1985, mientras realizaba un estudio ecológico dentro de la entonces Reserva Natural. También se tomaron muestras en áreas con bosque secundario en los alrededores del puesto de Boni Pérez, donde se encontraba el campamento, y en alrededores de Los Pirpintos (Protomastro, 1988).

Los especímenes de Parmeliaceae coleccionados, fueron depositados en BAFC. El colector fue citado en este trabajo, con sus iniciales (JJP) al mencionar el Material examinado, donde sólo se cita una selección de los especímenes estudiados. La abreviatura bv indica que la colección se hizo en bosque virgen. Las abreviaturas correspondientes a los nombres de los herbarios siguen el Index Herbariorum (Holmgren \& Holmgren, 2001). Los nombres de los autores de especies se abreviaron según Brummit \& Powell (1992).

Procedimientos de estudio de los especimenes $y$ datos de las especies. Los talos se examinaron bajo microscopio estereoscópico. La observación y medición de ascosporas y conidios se realizó examinando con microscopio óptico, cortes finos de apotecios y de talos, realizados a mano alzada, tratados con hidróxido de potasio $5 \%$ y luego teñidos con floxina acuosa al 1\%. Los metabolitos secundarios de importancia taxonómica se pusieron en evidencia de manera preliminar mediante reacciones de $\mathrm{K}$ (con hidróxido de potasio 10\%) y C (hipoclorito de sodio de uso doméstico, $20 \%$ aproximadamente) y $\mathrm{KC}$ (K seguido de C); la identificación definitiva se realizó mediante cromatografía en capa delgada (TLC, Culberson \& Ammann, 1979) por comparación con extractos acetónicos de especímenes cuyas sustancias eran conocidas (muestras patrón). En la mayoría de los casos se realizó una estimación de la cantidad relativa de cada metabolito secundario de importancia taxonómica, en base a la intensidad del color de cada mancha obtenida luego de la fase final del revelado (calentamiento a $110^{\circ} \mathrm{C}$ después del rociado de las placas con ácido sulfúrico al 10\% en agua) y su diámetro. Se usaron las siguientes categorías: mayor, intensidad fuerte del color y mancha de aproximadamente 8-10 mm diám.; submayor, intensidad mediana y mancha de 5-8 mm diám.; menor, intensidad de mancha mediana a leve y 3-5 mm diám.; trazas, intensidad muy suave y diámetro menor a $3 \mathrm{~mm}$. 


\section{T. Adler - Líquenes Parmelioides del Parque Nacional Copo}

\section{Resultados y Discusión}

Este trabajo describe por primera vez en conjunto, las especies parmelioides de la familia Parmeliaceae del Parque Nacional Copo, realizado en base al estudio de colecciones del año 1985 que constituye entonces un punto de partida en el tiempo para monitoreos de conservación.

\section{Descripción de las especies}

1- Bulbothrix subcoronata (Müll. Arg.) Hale, Phytologia 28: 481.1974.

Parmelia subcoronata Müll. Arg., Rev. Mycol. 9: 135. 1887.

Ilustraciones: Hale (1976a) p. 25, Fig. 7a; Ferraro (1981) p. 89, lámina 1, Fig. A.

Talo 2,0-6,0 cm diám., corticícola, gris claro, estrechamente adnato; lóbulos 1,0-4,0 mm de ancho, subirregulares alargados, ramificados de forma irregular, margen con cilias negras de base bulbosa. Superficie superior plana y lisa, emaculada, sin propágulos vegetativos. Médula blanca. Superficie inferior negra en el centro hasta castaño oscuro en los márgenes, moderadamente ricinada, ricinas concoloras, simples. Apotecios adnatos imperforados, 2,0-5,0 mm diám., anfitecio coronado de manera irregular, disco castaño, ascosporas hialinas elipsoides, 5,0-8,0 × 8,0-10,0 $\mu \mathrm{m}$. Picnidios con conidios bifusiformes $6,0-9,0 \mu \mathrm{m}$ de largo.

Química: Corteza superior $\mathrm{K}+$ amarillo (atranorina); médula $\mathrm{K}+$ amarillo tornándose anaranjado rojizo a rojo, $\mathrm{C}-, \mathrm{KC}-$ (ácido norstíctico, mayor).

Material examinado: Los Pirpintos, IV.1985, en bv, JJP s.n. (BAFC 36524), s/ Prosopis nigra, 27.V.1985, JJP s.n. (39235, 39242); Copo, en bv, VI.1985, JJP s.n. (BAFC 36523).

Obs.: Relativamente común en el área. Hale (1976a) la menciona para Brasil, Paraguay y Argentina (Misiones) considerándola una especie Sudamericana rara. Jungbluth et al. (2008) describen una especie nueva muy afín $(B$. regnelliana Jungbluth, Marcelli \& Elix) en base a la diferencia en el color de la superficie inferior: en el holotipo de $B$. subcoronata (único espécimen reconocido por ellos como perteneciente a esta especie) es descripta como de color negro brillante mientras que en $B$. regnelliana se describe como castaño claro a beige. Estos autores opinan que los especímenes mencionados por Hale (1976a), salvo el holotipo depositado en G, deben probablemente determinarse como $B$. regnelliana, a pesar de que no han examinado ningún material aparte del holotipo. Benatti (2012) discutió las diferencias de $B$. regnelliana con $B$. subcoronata (de la que dice conocer el holotipo, y haber examinado el espécimen Montes 10060 (MVM) antes determinado por Hale como B. subcoronata, e incluye este material en B. regnelliana. Según Benatti (2012) holo e isotipo de $B$. regnelliana, están en buenas condiciones pero es difícil encontrar ascosporas ya que los himenios a menudo carecen de ascos, aunque ambos especímenes tienen muchos apotecios.

Benatti además dice del holotipo de $B$. subcoronata (G), que consiste meramente de 4 fragmentos muy pequeños y que debido a la pobre condición del espécimen es difícil describirlo apropiadamente porque la mayor parte de los caracteres no pudieron ser observados; además sólo el himenio de un apotecio contiene ascosporas que son pocas, la mayoría de los ascos contiene una masa sin forma. Por esta razón el rango del largo de esporas medidos para el holotipo (G), no se puede comparar con el rango que Benatti da para $B$. regnelliana donde se citan muchos materiales examinados (Brasil, Paraguay, Argentina en Corrientes y Misiones). Estas dificultades con el holotipo de B. subcoronata, no permiten la observación de la variabilidad de la especie, por ejemplo del color del envés y del tamaño de las ascosporas, caracteres considerados importantes por Benatti en su discusión.

Dado el estado actual del conocimiento, parece razonable suponer que $B$. subcoronata es una especie más variable de lo aceptado por Jungbluth et al. y Benatti y por el momento aquí se adopta una visión amplia, en que los especímenes con envés castaño oscuro a mediano son considerados parte de la variabilidad de $B$. subcoronata.

Dentro de la Argentina, B. subcoronata fue citada anteriormente para Corrientes (Ferraro, 1981), además de Misiones. Aquí es citada por primera vez para Santiago del Estero.

2- Canoparmelia austroamericana Adler, Mycotaxon 28: 251. 1987.

Ilustraciones: Adler (1987) p. 252, Fig. 1; Adler (1988), p. 240, Fig. 6. 
Talo estrechamente adnato, corticícola o lignícola, 5,0-10,0 cm diám., lóbulos eciliados, con ápice subredondeado, irregularmente crenados. Superficie superior ligeramente maculada en partes jóvenes de los lóbulos. Sin propágulos vegetativos. Médula blanca. Superficie inferior beige a castaño claro, escasamente ricinada, ricinas simples, con una zona papilada a desnuda en el margen de los lóbulos. Apotecios abundantes, disco castaño, 2,0-8,0 mm diám., sin perforación central, ascosporas $6,0-8,0$ $\times 10,0-12,0 \mu \mathrm{m}$. Picnidios frecuentes, abundantes, inmersos, conidios bifusiformes a fusiformes, 5,0-7,0 $\mu \mathrm{m}$ de largo.

Química: Corteza superior $\mathrm{K}+$ (atranorina); médula $\mathrm{UV}-, \mathrm{K}-, \mathrm{C}-, \mathrm{KC}+$ rosado levemente violáceo (ácidos stenospórico, perlatólico y glomelliférico).

Material examinado: Los Pirpintos, V.1985, s/ Prosopis nigra, JJP s.n. (BAFC 35621); Copo, en bv, II.1985, JJP s.n. (BAFC 39321), en bv, VI.1985, JJP s.n. (BAFC 39320, 39363).

Obs.: Especie poco común en el área. Se la describió como especie nueva en base a material de Buenos Aires y Santiago del Estero (Adler, 1987) y hasta el momento se la conoce sólo para estas provincias en la Argentina, siendo considerada hasta 1998 como endémica de nuestro país. Quilhot et al. (1998) la citaron para Chile (Región I) donde parece ser rara. En la página liaslight.lias.net/ Description/ItemID_11499.html se establece que la especie no es sólo de América del Sur sino que estaría también presente en América Central, lo que es probablemente un error dado que este dato no fue documentado en ninguna publicación y tampoco fue citado el dato fehaciente en esta página, por esto se la incluye aquí en la categoría Endémica (endemismo del Sur de América del Sur).

3- Canoparmelia crozalsiana (de Lesd.) Elix \& Hale, Mycotaxon 27: 278. 1986.

Parmelia crozalsiana de Lesd. in Harmand, Lich. France 4: 555. 1910.

Pseudoparmelia crozalsiana (de Lesd.) Phytologia 28: 189. 1974.

Ilustraciones: Hale (1976b) p. 26, Fig. 10b, como Pseudoparmelia crozalsiana; Adler (1988), p. 241, Fig.8; Eliasaro (2001) p. 81, Fig. 25.

Talo gris a gris verdoso, hasta 10,0 cm diám., corticícola, adnato. Lóbulos con márgenes eciliados (0,5) 0,8-3,0 $\mathrm{mm}$ de ancho, crenados a incisos. Superficie superior con máculas reticuladas suaves en los lóbulos, sorediada, con soralios punctiformes, mayormente sobre crestas lineares, confluentes en la madurez. Médula blanca. Superficie inferior negra, moderada a densamente ricinada, con ricinas mayormente simples, excepto en una delgada zona marginal desnuda castaña bajo los lóbulos. Apotecios y picnidios no vistos.

Química: Corteza superior $\mathrm{K}+$ amarillo (atranorina); médula $\mathrm{K}+$ amarillo tornándose anaranjado claro (ácidos stíctico, mayor, menegazziaico, menor, constíctico, menor a trazas).

Material examinado: Los Pirpintos, IV.1985, JJP s. n. (BAFC 39322), VI. 1985, JJP, s.n. (BAFC 39280).

Obs.: Esta especie no es muy frecuente en el área de estudio. Recientemente Lendemer \& Hodkinson (2012) transfirieron el grupo de Parmelia crozalsiana (que incluye además a $P$. carneopruinata (Zahlbr.) Elix \& Hale, P. inhaminensis (Dodge) Elix \& Hale) y P. schelpei (Hale) Lendemer \& Hodkinson, al género Crespoa (D. Hawksw.) Lendemer \& Hodkinson, basado en Parmotrema subgénero Crespoa D. Hawksw.; en el presente trabajo se prefirió mantener el nombre Canoparmelia para el género por ser el más utilizado en los últimos años.

Según Hale (1976b) y Elix (1994) la especie está presente en América del Norte y del Sur, en el sur de Europa, sur y este de África, India y Australia, por lo tanto se la incluye en la categoría Cosmopolita (Galloway, 2008).

En Argentina fue citada para Tucumán, Misiones, Córdoba y Buenos Aires (Estrabou \& Adler, 1999), pero se la conoce también para otras provincias particularmente del NOA, como ser Salta y Jujuy (Adler, datos no publicados). Ésta es la primera cita para Santiago del Estero.

4- Canoparmelia texana (Tuck.) Elix \& Hale, Mycotaxon 27: 279. 1986.

Parmelia texana Tuck. Amer. J. Arts, ser. 2, 25: 424. 1858.

Pseudoparmelia texana (Tuck.) Hale, Phytologia 28: 191. 1974.

Ilustraciones: Hale (1976b) p. 51, Fig. 17f, como Pseudoparmelia texana; Adler (1988), p. 242, Fig. 9; Elix (1994) p. xii, Fig. 5. 


\section{T. Adler - Líquenes Parmelioides del Parque Nacional Copo}

Talo gris verdoso a gris, hasta $10,0 \mathrm{~cm}$ diám., corticícola. Lóbulos alargados con ápices subirregulares a redondeados, eciliados, 1,5-6,0 $\mathrm{mm}$ de ancho. Superficie superior ligeramente maculado-reticulada con máculas lineares blancas en los lóbulos jóvenes, con fisuras en las zonas más maduras o viejas. Sorediada, con soralios punctiformes a orbiculares principalmente sobre pliegues de la superficie, coalesciendo en la madurez. Médula blanca. Superficie inferior negra, escasamente ricinada, ricinas negras generalmente simples, y una angosta franja marginal castaña desnuda. Apotecios y picnidios no vistos.

Química: Corteza superior $\mathrm{K}+$ amarillo (atranorina); médula $\mathrm{K}-, \mathrm{C}-, \mathrm{KC}-$, (ácidos divaricático, mayor, nordivaricático, mayor).

Material examinado: Los Pirpintos, IV. 1985, JJP s.n. (BAFC 39244); s/Prosopis nigra, 27.V.1985, JJP s.n. (BAFC 39243), s/Aspidosperma quebracho-blanco, JJP s.n. (BAFC 39324); Copo, en bv, VI.1985, JJP s.n. (BAFC 39323, 39362).

Obs.: Hale (1976b) la citó para América del Norte, Central y del Sur, África del Sur y Este, India, Japón y Tailandia. Elix (1994) la citó para Australia. Según estos autores esta especie pertenece a las categorías Pantemplada (aunque ausente en Europa) y Pantropical (a elevadas alturas). Osorio \& Ferraro (1975) la citaron para Corrientes, conociéndosela también para Buenos Aires, Entre Ríos y Córdoba (Estrabou \& Adler, 1999). Es conocida también para otras provincias, particularmente del NOA, p. ej. Salta y Jujuy (Adler, datos no publicados). Ésta es la primera mención para Santiago del Estero.

5- Hypotrachyna aspera, C. H. Ribeiro \& Marcelli, Mitt. Inst. Allg. Bot. Hamburg 30-32: 133. 2002.

Ilustración: Marcellli \& Ribeiro (2002) p.134, Fig. 6.

Talo hasta $5,0 \mathrm{~cm}$ diám, corticícola o saxícola. Lóbulos sublineares, 1,0-3,0 $\mathrm{mm}$ de ancho, subdicotómicos. Superficie superior emaculada, con dáctilos irregulares huecos, cerrados hasta abiertos apicalmente y a veces sorediados con soredios granulares. Médula blanca, con pigmento amarillo K+ púrpura bajo los dáctilos. Superficie inferior negra, moderadamente ricinada con ricinas dicotómicas concoloras con pocas ramificaciones. Apotecios y picnidios no vistos.
Química: Corteza superior $\mathrm{K}+$ amarillo (atranorina); médula $\mathrm{K}+$ amarillo tornándose rojo amarronado, $\mathrm{C}-, \mathrm{KC}-\mathrm{o} \mathrm{KC}+$ rosado (ácidos colensoico, mayor, norcolensoico, mayor, hidroxicolensoico, submayor, y un pigmento amarillo $\mathrm{K}+$ púrpura, skyrina, menor).

Material examinado: Los Pirpintos, 20.II.1986, corticícola, JJP s.n. (BAFC 39272), Copo, en bv, VI.1985, JJP s.n. (BAFC 36516, 36517, 36518, 36716), en bv, X. 1985 (36515).

Obs.: El material aquí mencionado para Copo, fue primeramente publicado bajo el nombre Hypotrachyna immaculata (Kurok.) Hale por Adler \& Elix (1992), como cita nueva de esta especie para Santiago del Estero. Luego de una nueva identificación con revisión de la química del material estudiado y de su morfología, se llegó a la conclusión de que el presente material corresponde a $H$. aspera Marcelli \& Ribeiro que carece de soralios primarios, característica morfológica de $H$. immaculata. H. aspera es también saxícola según Marcelli \& Ribeiro (2002).

Sipman et al. (2009) establecieron las principales diferencias entre tres especies de Hypotrachyna muy cercanas entre sí, con superficie dáctilo-pustulada y médula con compuestos del grupo colensoico-livídico: H. pustulifera (Hale) Skorepa (ancho de lóbulos 2,0-7,0 mm), H. aspera Ribeiro \& Marcelli (lóbulos 1,0-2,5 mm) y $H$. subpustulifera Elix (ancho de lóbulos 0,5-1,0 mm). $\mathrm{Si}$ bien las tres especies contienen como sustancias medulares compuestos del grupo colensoicolivídico, hay diferencias que correlacionan con el ancho de los lóbulos, particularmente la ausencia de varios compuestos en $H$. aspera, como ser el ácido livídico, y los ácidos metoxicolensoico, fisódico y 4-O-metilfisódico entre otros, mientras que el pigmento amarillo en $H$. pustulifera no es skyrina sino pigmentosina $\mathrm{A}$, además de la ausencia de pigmentos medulares en $H$. subpustulifera.

Según Sipman et al. (2009) H. aspera era conocida sólo para Brasil (estados de Minas Gerais y Paraná), por lo que ésta es la primera mención fuera de Brasil, y la primera para Argentina y Santiago del Estero, lo que constituye una importante extensión de la distribución hacia el Sur, que permite incluirla tentativamente en la categoría Sudamericana.

6- Myelochroa lindmanii (Lynge) Elix \& Hale, Mycotaxon 29: 41. 1987. 
Parmelia lindmanii Lynge, Ark. Bot. 13: 74. 1914.

Parmelina lindmanii (Lynge) Hale, Smithsonian Contr. Bot. 33: 36. 1976.

Ilustraciones: Hale (1976c) p. 33, Fig. 16f, como Parmelina lindmanii; Adler (1988) p. 247, Fig. 19; Eliasaro (2001) p. 137, Fig. 53.

Talo adnato, gris oliváceo, hasta 5,0 cm diám., corticícola. Lóbulos subirregulares, apicalmente subredondeados, 2,0-4,0 $\mathrm{mm}$ de ancho, con cilias muy escasas, muy cortas, menos de $0,5 \mathrm{~mm}$ en los márgenes en las axilas de los lóbulos. Superficie superior con isidios globosos a cilíndricos, pequeños, hasta $0,2 \mathrm{~mm}$ de alto. Médula amarilla. Superficie inferior negra con ricinas concoloras, con una franja castaña marginal desnuda bajo los lóbulos. Apotecios y picnidios no vistos.

Química: Corteza superior $\mathrm{K}+$ amarillo (atranorina); médula $\mathrm{K}+, \mathrm{C}+$ y $\mathrm{KC}+$ amarillo más intenso que el color de la médula (ácidos secalónicos, mayores).

Material examinado: Copo, X.1985, corticícola s/rama en bv, JJP s.n. (BAFC 39327B).

Obs.: Esta especie se cita aquí como perteneciente al género Myelochroa, a pesar de que en un trabajo reciente (Eliasaro et al., 2010) se llegó a la conclusión de que no pertenecería al mismo sino a Parmelinella, ambos delimitados por Elix \& Hale (1987). A pesar de los resultados de dicha comparación molecular, los autores no realizaron una nueva combinación de manera formal por estimar necesarios más estudios.

Es una especie de distribución Americana, citada para México y América del Sur, en Colombia, Brasil, Venezuela, Paraguay, Uruguay, Argentina (Hale, 1976c). En la página liaslight.lias.net/Description/ ItemID_8086.html se establece que la especie no es sólo de América del Norte y del Sur sino que está también presente en América Central. En Argentina fue citada para Misiones (Osorio 1969), Buenos Aires (Osorio, 1970, Adler, 1992), Corrientes (Ferraro, 1981) y Córdoba (Estrabou et al., 2005). Es rara en el área de estudio siendo ésta la primera mención para Santiago del Estero.

7- Parmelinopsis bonariensis Adler \& Elix, Mycotaxon 30: 341. 1987.

Ilustraciones: Adler \& Elix (1987) p. 343, Fig. 3; Adler (1988) p. 248, Fig. 22.
Talo corticícola, laxamente adnato, gris claro, hasta 3,0 cm diám.; lóbulos 1,0-2,0 mm de ancho, apicalmente truncados, cilias marginales conspicuas en las axilas de los lóbulos, algunas orientadas hacia el sustrato, sin isidios, soredios ni pústulas. Superficie superior emaculada o muy débilmente maculada. Médula blanca. Superficie inferior negra hasta parda, ricinas concoloras, predominantemente simples, a veces también bifurcadas. Apotecios subestipitados, ascosporas 5,0-8,0 × 9,5-13,0 $\mu \mathrm{m}$. Picnidios con conidios bifusiformes, $6,0-6,6 \mu \mathrm{m}$ de largo.

Química: Corteza superior $\mathrm{K}+$ amarillo (atranorina); médula $\mathrm{K}-, \mathrm{C}-, \mathrm{KC}+$ rosado violáceo, tornándose anaranjado-rojizo (ácidos 3-metoxi2,4-di- $O$-metilgirofórico, mayor, girofórico, mayor, umbilicárico, menor, hiáscico, trazas y 3-metoxiumbilicárico, trazas).

Material examinado: Copo. II.1985, corticícola, JJP s.n. (BAFC 36521).

Obs.: Especie rara en el Parque Nacional Copo, hasta ahora parece ser Endémica de Argentina. Se la describió como especie nueva en base a especímenes saxícolas muy bien desarrollados en la provincia de Buenos Aires (Adler \& Elix, 1987), donde también se coleccionaron algunos ejemplares corticícolas pequeños. Aparentemente el sustrato preferencial es la roca, aunque crece también sobre corteza. Fue citada anteriormente para Santiago del Estero por Adler \& Elix (1992).

8- Parmotrema andinum (Müll. Arg.) Hale, Phytologia 28: 334. 1974.

Parmelia andina Müll. Arg., Rev. Mycol. 1: 169. 1870.

Ilustraciones: Swinscow \& Krog (1988) p. 167, Fig. 84; Adler (1988) p. 249, Fig. 23; Popoff \& Ferraro (2002) p. 407, Fig. 7.7A.

Talo gris a gris verdoso, corticícola, hasta $10,0 \mathrm{~cm}$ diám., laxamente adherido al sustrato. Lóbulos subirregulares con ápices subredondeados, 13,0-15,0 $\mathrm{mm}$ de ancho, márgenes eciliados. Superficie superior emaculada, sin soredios, isidios, ni pústulas. Médula blanca. Superficie inferior negra en el centro, escasamente ricinada con ricinas gruesas, y una franja marginal ancha desnuda de color castaño a castaño claro hasta marfilino. Apotecios frecuentes, abundantes, estipitados; disco 


\section{T. Adler - Líquenes Parmelioides del Parque Nacional Copo}

castaño hasta 15,0 mm diám., con o sin perforación central y el margen del anfitecio liso, sin cilias; ascosporas 6,5-8,0 $(10,0) \times 11,5-14,0(17,0) \mu \mathrm{m}$. Picnidios agrupados en áreas submarginales de los lóbulos, abundantes. Conidios filiformes $(11,0)$ $13,0-19,5 \times 0,5 \mu \mathrm{m}$.

Química: Corteza superior $\mathrm{K}+$ (atranorina); médula $\mathrm{K}-, \mathrm{C}+$ rojo oscuro, $\mathrm{KC}+$ rojo oscuro tornándose anaranjado (ácido lecanórico, mayor).

Material examinado: Copo, corticícola, VI.1985 JJP s.n. (BAFC 39257, 39258), VIII.1985 (BAFC 39256, 39261), en bv, IX.1985 JJP s.n. (BAFC 39254, 39259, 39325), en bv, X.1985 JJP s.n. (BAFC 39326), s/Schinopsis lorentzii, X.1985 (BAFC 39255).

Obs.: Parmotrema andinum es la contraparte sin propágulos vegetativos de $P$. austrosinense (Zahlbr.) Hale. Es común en el área de estudio. Según Hale (1965) se la registró para América del Sur y África-Madagascar, por lo que se la conoció por mucho tiempo como especie SudamericanaAfricana (Galloway, 2008), hasta que fue citada para China por Chen et al. (2005), por lo que ahora se define como Afro-Sudamericana-asiática, al igual que P. yodae (Adler \& Calvelo, 2010).

En la Argentina había sido mencionada para Salta (Hale, 1965), Buenos Aires (Osorio \& D'Espósito, 1979; Adler 1992), Jujuy (Osorio \& Ferraro, 2001) y Corrientes (Popoff \& Ferraro, 2002). Ésta es la primera cita para Santiago del Estero.

9- Parmotrema argentinum (Kremp.) Hale, Phytologia 28: 334. 1974.

Parmelia argentina Kremplh., Flora 61: 476. 1878.

Ilustraciones: Hale (1965) pl. 15, Fig. 43, como Parmelia argentina; Canêz (2005) p. 257 Fig. 45; Donha (2005) p. 38, Fig. 8; Popoff \& Ferraro (2002) p. 407, Fig. 7.7B.

Talo hasta $10,0 \mathrm{~cm}$ diám., flojamente adnato, corticícola, gris a gris verdoso. Lóbulos subirregulares, apicalmente redondeados, 5,0-15,0 $\mathrm{mm}$ de ancho, suberectos, márgenes lisos a crenados, ciliados, cilias principalmente simples $\mathrm{K}+$ violeta, hasta $2,5 \mathrm{~mm}$ de largo. Superficie superior emaculada hasta ligeramente maculada en el centro del talo. Médula blanca. Superficie inferior negra en el centro con ricinas negras, con una ancha y bien delimitada franja blanca desnuda bajo los lóbulos. Apotecios frecuentes, estipitados, disco castaño, 5,0-12,0 mm diám., imperforado, margen con dientes cortos a escasamente ciliado. Ascosporas 6,5-10,0 (11,0) × 13,0-16,5 $(18,0)$ $\mu \mathrm{m}$. Picnidios frecuentes, abundantes, en áreas submarginales del talo; conidios filiformes cortos $(6,0) 7,0-8,0(9,0) \times 0,5 \mu \mathrm{m}$.

Química: Corteza superior $\mathrm{K}+$ amarillo (atranorina, mayor a menor); médula UV+ celeste, $\mathrm{K}-, \mathrm{C}-, \mathrm{KC}+$ rojo violáceo, volviéndose anaranjado (ácidos alectorónico, mayor, $\alpha$-colatólico, mayor a menor y un compuesto no identificado relacionado con el ácido alectóronico).

Material examinado: Los Pirpintos, corticícola, IV.1985 JJP s.n. (BAFC 39239); s/ Aspidosperma quebracho-blanco y Prosopis nigra, 27.V.1985, JJP s.n. (BAFC 39260); Copo, s/ ramas en bv, II.1985, JJP s.n. (BAFC 39262), VI.1985, JJP s.n. (BAFC 39263), VIII.1985 (BAFC 39240).

Obs.: Es bastante frecuente en el área de estudio. Según Hale (1965) P. argentinum se distribuye por México, Honduras, Venezuela, Brasil, Paraguay y Argentina (provincia de Salta, Río Pescado). Para Bolivia fue citada recientemente por Kukwa et al. (2012). Una cita anterior para China, Taiwan (Kurokawa \& Lai, 2001), indica que su distribución puede ser amplia y se la podría caracterizar como Americana-Asiática. Fue citada también en Argentina por Osorio (1981) para Misiones y para Corrientes por Popoff \& Ferraro (2002). Aquí es citada por primera vez para Santiago del Estero.

10- Parmotrema austrosinense (Zahlbr.) Hale, Phytologia 28: 335. 1974.

Parmelia austrosinensis Zahlbr., Symb. Sin. 3: 192. 1930.

Ilustraciones: Swinscow \& Krog (1988) p. 169, Fig. 85; Adler (1988) p. 24, Fig. 24; Popoff \& Ferraro (2002) p. 407, Fig. 7.7C.

Talo hasta $8,0 \mathrm{~cm}$ diám, gris claro, corticícola, laxamente adnato, fijado en el centro. Lóbulos suberectos, redondeados, 3,0-10,0 $\mathrm{mm}$ de ancho, márgenes eciliados, crenados, sorediados, soralios punctiformes, semilunares, o lineares. Superficie superior emaculada a muy ligeramente maculada. Médula blanca. Superficie inferior negra en el 
centro con escasas ricinas negras simples, y una ancha franja marginal desnuda castaña clara a marfilina bajo los lóbulos. Apotecios y picnidios no vistos.

Química: Corteza superior $\mathrm{K}+$ amarillo (atranorina); médula $\mathrm{K}-, \mathrm{C}-, \mathrm{KC}+$ rojo violáceo tornándose anaranjado (ácido lecanórico, mayor).

Material examinado: Los Pirpintos, A) s/ Aspidosperma quebracho-blanco, B) s/Prosopis nigra, 27.V.1985, JJP s.n. (BAFC 39329); Copo, corticícola, 20.II.1985, JJP s.n. (BAFC 39330), X.1985, corticícola en bv, JJP s.n. (BAFC39327A, 39327'), en bv, VI.1985, JJP s.n. (BAFC 39328).

Obs.: Es común en el área de estudio. Desde el punto de vista biogeográfico se la puede incluir en la categoría Pantropical caracterizada ya por Hale (1965) estando presente también en áreas templadas adyacentes (Louwhoff, 2001; Nash \& Elix, 2002). Recientemente fue citada para Taiwan por Kurokawa \& Lai (2001). En la Argentina fue citada para Jujuy, Salta, Tucumán, La Rioja, Córdoba, Corrientes y Buenos Aires (Adler \& Calvelo, 2010) y en particular para Santiago del Estero, Dpto. Copo, Los Pirpintos, por Osorio \& Ferraro (1976). Es una especie frecuentemente coleccionada en el país.

11- Parmotrema cetratum (Ach.) Hale, Phytologia 28: 335. 1974.

Parmelia cetrata Ach.. Syn. Lich. 198. 1814.

Lista de sinónimos en Hale \& Fletcher, 1990.

Ilustraciones: Adler (1988) p. 250, Fig. 25; Popoff \& Ferraro (2002) p. 407, Fig. 7.7D; Benatti \& Marcelli (2008) p. 78, Figs. 1a y 1 b.

Talo gris a gris verdoso, adnato a laxamente adnato, hasta 10,0 cm diám., corticícola o lignícola. Lóbulos subirregulares frecuentemente con ápices subredondeados, 3,0-5,0 $\mathrm{mm}$ de ancho, crenados a incisos, en partes algo laciniados, lacinias cortas, escasa a moderadamente ciliadas o algunos lóbulos sin cilias, cilias $0,2-1,5 \mathrm{~mm}$ de largo, mayormente simples, raramente escuarrosas o bifurcadas. Sin soredios, isidios ni pústulas. Superficie superior con máculas reticulares o con puntos verdes sobre fondo gris y fisuras desarrolladas de manera reticular. Médula blanca. Superficie inferior negra con ricinas negras simples a bifurcadas o escuarrosas, esparcidas o densamente dispuestas, color castaño a pardo oscuro en una franja marginal bajo los lóbulos, desnuda o moderada a densamente ricinada hasta los márgenes, con ricinas mayormente simples. Apotecios con disco castaño, 3-15 mm diám., generalmente sin perforación central aunque los maduros pueden ser perforados; ascosporas 6,7-7,5 $\times 10,0-11,4 \mu \mathrm{m}$. Picnidios frecuentes, agrupados en áreas submarginales de lóbulos y lacinias. Conidios filiformes, $11,0-14,0 \times 0,5 \mu \mathrm{m}$.

Química: Corteza superior $\mathrm{K}+$ amarillo (atranorina, minor); médula $\mathrm{K}+$ amarillo tornándose rojo oscuro (ácidos salacínico, mayor, consalacínico, menor).

Material examinado: Los Pirpintos, s/Prosopis nigra, 20.II.1985, JJP s.n. (BAFC 39354, 39358), s/Aspidosperma quebracho-blanco, 27.V.1985, JJP s.n. (BAFC 39355); Copo, s/Aspidosperma quebracho-blanco, II.1985, JJP s.n. (BAFC 39290); corticícola en bv, VI.1985, JJP s.n. (BAFC 39285), en bv, X.1985 (BAFC 39291, 39357), en el puesto de Boni Pérez, lignícola s/poste de quebracho colorado, XII.1985, JJP s.n. (BAFC 39289, 39348), II.1986, JJP s.n. (BAFC 39347).

Obs.: Esta especie es común en el área de estudio. La superficie superior aquí suele presentar un punteado verde sobre fondo gris además de una maculación reticular, debido posiblemente a intensa exposición al sol. Es una especie con distribución Pantemplada y Pansubtropical (Hale \& Fletcher, 1990). Es coleccionada muy frecuentemente en el país.

12- Parmotrema conferendum Hale, Mycotaxon 5: 433.1977.

Rimeliella conferenda (Hale) Kurokawa, Ann. Tsukuba Bot. Gard. 10: 4. 1991.

Canomaculina conferenda (Hale) Elix, Mycotaxon 65: 476. 1997.

Ilustraciones: Hale (1977) p. 442, Fig. 2; Adler (1988) p. 251, Fig. 26; Canêz (2005) p. 250, Fig. 4, como Canomaculina conferenda.

Talo gris claro hasta $5,0 \mathrm{~cm}$ diám., laxamente adherido al sustrato, corticícola. Lóbulos 0,5-1,0 cm de ancho, más o menos ascendentes, apicalmente redondeados, márgenes ondulados y cortamente ciliados que pueden faltar en algunas partes. Superficie con maculación efigurada, sorediada predominantemente en los márgenes y 


\section{T. Adler - Líquenes Parmelioides del Parque Nacional Copo}

áreas submarginales. Médula blanca. Superficie inferior con ricinas dimórficas, negra en el centro, con ricinas gruesas y largas y una zona marginal castaño claro a marfilino en el resto de la superficie, densamente ricinada con ricinas delgadas y cortas. Apotecios y picnidios no vistos.

Química: Corteza superior $\mathrm{K}+$ amarillo (atranorina); médula $\mathrm{K}-, \mathrm{C}-, \mathrm{KC}+$ rojo violáceo virando al anaranjado (norlobaridona, mayor, loxodina, menor).

Material examinado: Copo, corticícola en bv, VI.1985, JJP s.n. (BAFC 39331).

Obs.: Esta especie es rara en el área. Fue descripta por Hale (1977) para elevar al nivel de especie una de las cepas químicas (con norlobaridona y loxodina) de $P$. subsumptum y fue citada por este autor para América del Norte (EEUU y México), América Central (Guatemala) y del Sur (Venezuela y Argentina, en Tucumán), siendo según él una especie americana poco frecuente. Krog \& Swinscow (1981) la mencionaron para el este de África bajo el nombre de Parmelia subsumpta Lynge, cepa química con norlobaridona y loxodina, siendo aparentemente rara allí también. Eliasaro \& Donha (2003) la citaron para el estado de Paraná y Canêz (2005) para el estado de Rio Grande do Sul (Brasil). Se la caracteriza como una especie con distribución AmericanaAfricana (Adler \& Calvelo, 2010). En Argentina fue también citada para la provincia de Buenos Aires por Adler (1988, 1992), donde parece ser una especie bastante común así como en Corrientes (Popoff \& Ferraro, 2002) donde se la citó con el nombre de $P$. subsumptum. Aquí se la menciona por primera vez para Santiago del Estero.

13- Parmotrema consors (Nyl.) Krog \& Swinscow, Lichenologist 15: 129. 1983.

Parmelia consors Nyl., Flora 68: 613. 1885.

Parmelina consors (Nyl.) Hale, Smithsonian Contr. Bot 28: 482.1974.

Canomaculina consors (Nyl.) Elix \& Hale, Mycotaxon 19: 239. 1987.

Ilustraciones: Hale (1976c) p. 21, Fig. 11d, como Parmelina consors; Adler (1988) p. 238, Fig. 2; Elix (1994) p. xi, Fig. 4; Eliasaro (2001) p. 68, Fig. 18.

Talo adnato a laxamente adnato, gris verdoso, coriáceo, hasta $5,0 \mathrm{~cm}$ diám., corticícola o lignícola. Lóbulos 2,0-5,0 mm de ancho, crenados, notoriamente ciliados, cilias gruesas, acuminadas, en la axilas de los lóbulos, hasta 1,0 mm de largo, simples a raramente bifurcadas, frecuentemente muy cortas. Superficie superior con notoria maculación efigurada a punteada de blanco, sin propágulos vegetativos. Médula blanca. Superficie inferior negra en el centro, con una franja color castaño oscuro en el margen, ricinas dimórficas, gruesas y largas en el centro, más cortas y delgadas en el resto de la superficie inferior. Apotecios frecuentes, adnatos a cortamente estipitados, disco castaño hasta $6,0 \mathrm{~mm}$ diám., generalmente imperforado a veces con alguna perforación, margen liso a crenado. Ascosporas 8,7-10,7 × 13,0-15,0 $\mu \mathrm{m}$. Picnidios frecuentes, conidios filiformes $11,0-14,0$ $\times 0,5 \mu \mathrm{m}$.

Química: Corteza superior $\mathrm{K}+$ amarillo (atranorina); médula $\mathrm{K}-, \mathrm{C}-, \mathrm{KC}-$ (sustancias no demostradas por TLC).

Material examinado: Los Pirpintos, s/ Aspidosperma quebracho-blanco, 20.II.1985, JJP s.n. (BAFC 39276, 39332); Copo, en bv, X.1985, JJP s.n. (BAFC 39269).

Obs.: Especie bastante común en el área de estudio. Hale (1976c) la citó para Brasil, Uruguay y la Argentina (Misiones y Buenos Aires) y Elix (1994) la citó para Australia, donde parece ser rara. Por lo tanto se la caracteriza como SudamericanaAustralásica (Adler \& Calvelo, 2002). En la Argentina fue citada para Entre Ríos por Osorio (1975) y Corrientes por Ferraro (1981). Ésta es la primera cita para Santiago del Estero.

14- Parmotrema cristobaliae (Ferraro \& Elix) O. Blanco, A. Crespo, Divakar, Elix \& Lumbsch, Mycologia 97: 157. 2005.

Rimeliella cristobaliae Ferraro \& Elix, Mycotaxon 49: 406. 1993. CTES-Holotipo!

Canomaculina cristobaliae (Ferraro \& Elix) Elix, Mycotaxon 65: 477. 1997.

Ilustraciones: Ferraro \& Elix (1993) p. 407, Fig. 2, como Rimeliella cristobalii; Spielmann (2009) p. 119, Fig. 18.

Talo corticícola o lignícola, folioso, gris a gris verdoso, laxamente adnato al sustrato, hasta $10 \mathrm{~cm}$ diám., sin soredios, isidios ni pústulas. Lóbulos subirregulares, 5,0-10,0 mm de ancho, márgenes con 
cilias esparcidas, simples a bifurcadas, hasta 1,0 mm de largo, frecuentemente con ricinas marginales que se pueden confundir con cilias, y a veces eciliados. Lóbulos crenados a incisos hasta profundamente incisos a laciniados, lacinias simples y cortas, 0,2 $\mathrm{mm}$ de largo hasta largas $(5,0-10,0 \mathrm{~mm})$ y muy divididas. Superficie superior con máculas blancas reticulares que suelen fisurarse, o con puntos verdes sobre fondo gris. Médula blanca. Superficie inferior negra, moderada a densamente ricinada hasta los márgenes o a veces con una franja desnuda, ancha o angosta, parda o castaña hasta raramente beige, en los márgenes de lóbulos o lacinias; ricinas negras, frecuentemente simples hasta ramificadas de manera irregular, a veces escuarrosas, 0,25-1,5 $(2,0) \mathrm{mm}$ de largo. Apotecios frecuentes, generalmente cupuliformes, estipitados, disco castaño con o sin perforación central, margen liso, eciliado, anfitecio notoriamente maculado. Ascosporas hialinas elipsoidales, (6) $8,5(11,5) \times 10,0-13,0$ $\mu \mathrm{m}$, episporio $0,5-1,5 \mu \mathrm{m}$. Picnidios frecuentes, inmersos en el talo en áreas submarginales de los lóbulos o puntas de las lacinias. Conidios filiformes $(8,0) 10-11(13) \times 0,5 \mu \mathrm{m}$.

Química: Corteza superior $\mathrm{K}+$ amarillo (atranorina); médula $\mathrm{K}+$ amarillo tornándose rojo oscuro, $\mathrm{C}-, \mathrm{KC}-$ (ácidos salacínico, mayor y consalacínico, menor a trazas, norlobaridona, mayor a menor, loxodina, menor a trazas).

Material examinado: Los Pirpintos, IV.1985, JJP s.n. (BAFC 39352). Copo, Puesto de Boni Pérez, en bv, XII.1985, J. J. P s.n. (BAFC 39292, CTES); Copo, II.1986 (BAFC 39293), VI.1985, JJP s.n. (BAFC 39350, 39353), IX.1985, JJP s.n. (BAFC 39349, 39356).

Obs.: Parmotrema cristobaliae fue descripta por Ferraro \& Elix (1993) como perteneciente al género Rimeliella Kurok., donde fue ubicada debido a una errónea interpretación del tipo de maculación de la superficie superior así como del tipo de ricinas: la primera no es realmente efigurada sino una variación del tipo reticulada con grietas sobre las máculas, típicas de Rimelia Hale \& Fletcher (1990) y las ricinas no son típicamente dimórficas según fueron descriptas por Kurokawa (1991) para Rimeliella. Para la diagnosis no se describió la presencia de ricinas escuarrosas (típicas de Rimelia) bien visibles en el holotipo y en los especímenes del Parque Nacional Copo estudiados aquí. La misma interpretación de la diagnosis se repitió en trabajos posteriores (Elix, 1997; Spielmann, 2009). Otro carácter que acerca esta especie al grupo de las incluidas en Rimelia es la frecuencia de lacinias.

En resumen, esta especie se caracteriza por los lóbulos crenados y frecuentemente laciniados, con cilias esparcidas cortas, y en algunos lóbulos ausentes, por la carencia de propágulos vegetativos, por la superficie superior con maculación y fisuración reticular, la superficie inferior mayormente negra, la presencia de ricinas escuarrosas entremezcladas con las simples y las irregularmente ramificadas y por la presencia de norlobaridona y loxodina además de los ácidos salacínico y consalacínico en la médula.

En la clave final se la considera tanto ciliada como no ciliada para facilitar la identificación, dado que porciones importantes de algunos especímenes pueden ser mayormente eciliadas.

Aquí se considera a $P$. cristobaliae como estrechamente relacionada a $P$. cetratum, de la cual se puede distinguir principalmente por la química medular: P. cristobaliae tiene norlobaridona y loxodina, además de ácidos salacínico y consalacínico, como sustancias medulares mayoritarias mientras $P$. cetratum presenta sólo estos dos últimos compuestos.

En el área de estudio las colecciones de $P$. cetratum difieren morfológicamente de las de $P$. cristobaliae en que la primera tiene los talos más adnatos y menos laciniados que la segunda.

Otra especie cercana es $P$. afrocetratum (Elix et al., 2005), con química medular idéntica a $P$. cetratum ( $\sin$ norlobaridona ni loxodina) pero $\sin$ cilias en los lóbulos y con conidios sublageniformes en vez de filiformes largos.

$P$. cristobaliae podría interpretarse como un híbrido entre $P$. cetratum y $P$. homotomum, que también carece de propágulos vegetativos y que produce norlobaridona y loxodina medulares en vez de ácido salacínico. $P$. homotomum no ha sido coleccionada hasta ahora en el área de estudio. La inclusión dentro de Parmotrema A. Massal. resultó de un estudio molecular (Blanco et al., 2005).

P. cristobaliae, fue hasta ahora registrada por Ferraro \& Elix (1993) sólo para Bolivia y Argentina (Chaco y Corrientes). Aquí se la menciona por primera vez para Santiago del Estero, siendo Endémica del Sur de Sudamérica. 


\section{T. Adler - Líquenes Parmelioides del Parque Nacional Copo}

15- Parmotrema hababianum (Gyeln.) Hale, Phytologia 28: 336. 1974.

Parmelia hababiana Gyel., Rep. Nov. Spec. Regni veg. 29: 288. 1931.

Ilustraciones: Hale (1965) pl. 15, Fig. 43, como Parmelia hababiana; Internet, Encyclopedia of Life (eol. org) con acceso a varias imágenes de Smithsonian Institution.

Talo laxamente adnato, corticícola sobre ramas jóvenes, hasta $5,0 \mathrm{~cm}$ diám., gris a gris verdoso. Lóbulos suberectos, márgenes crenados, escasamente ciliados, cilias hasta $1 \mathrm{~mm}$ de largo, sorediados, soralios capitiformes, semilunares a lineares, extendiéndose submarginalmente. Superficie superior emaculada a ligeramente maculada. Médula blanca. Superficie inferior negra en el centro, escasamente ricinada, las ricinas simples o irregularmente ramificadas, con una ancha franja marginal desnuda, castaña clara hasta marfilina. Apotecios y picnidios no vistos.

Química: Corteza superior $\mathrm{K}+$ amarillo (atranorina); médula $\mathrm{K}-, \mathrm{C}-, \mathrm{KC}-$ (ácido protoliquesterínico, mayor).

Material examinado: Copo, VI.1985, corticícola s/rama joven, JJP s.n. (BAFC 39333).

Obs.: Esta especie es rara en el área de estudio. Aquí se incluyen sólo los ejemplares de la cepa con ácido protoliquesterínico (Krog \& Swinscow, 1981) dado que los ejemplares con norlobaridona fueron incluidos por Hale (1977) en P. yodae.

La cepa con ácido protoliquesterínico fue citada por Hale (1965) para México y diferentes localidades de África, lo que indica una distribución AmericanaAfricana (Adler \& Calvelo, 2007), aunque es posible que el número de especímenes de esta cepa haya sido coleccionada en defecto y los datos no reflejen su verdadera distribución. Nash \& Elix (2002) citan a $P$. hababianum para Sonora (México), pero describieron la cepa con norlobaridona que aquí es llamada P. yodae (Kurok.) Hale. En la Argentina $P$. hababianum fue citada por Estrabou \& Adler (1999) por primera vez para Córdoba. Ésta es la primera vez que se la cita para Santiago del Estero.

16- Parmotrema mesotropum (Müll. Arg.) Hale, Phytologia 28: 337. 1974.

Parmelia mesotropa Müll. Arg. Rev. Mycol. 10: 55. 1888.
Ilustraciones: Eliasaro (2001) p. 176, Fig. 72; Popoff \& Ferraro (2002) p. 408 Fig. 7.8B; Internet, Encyclopedia of life (eol. org).

Talo gris mineral, adnato, hasta $10,0 \mathrm{~cm}$ diám., corticícola. Lóbulos 3,0-8,0 mm de ancho, subirregulares conápices subredondeados, crenados, eciliados. Superficie superior muy arrugada en el centro, emaculada. Médula blanca. Superficie inferior totalmente negra, moderada a densamente ricinada, con una angosta franja marginal parda desnuda. Apotecios frecuentes, abundantes, con disco pardo, hasta $8,0 \mathrm{~mm}$ diám., con margen liso a crenado, mayormente sin perforación central. Ascosporas $(8,0) 10,0-11,5 \times 14,5-20,0(21,0) \mu \mathrm{m}$. Picnidios frecuentes, en áreas submarginales de los lóbulos; conidios sublageniformes, 7,0-8,5 × 0,5 $\mu \mathrm{m}$.

Química: Corteza superior $\mathrm{K}+$ amarillo (atranorina); médula $\mathrm{K}-\mathrm{o} \mathrm{K}+$ amarillo pálido, $\mathrm{C}-, \mathrm{KC}-$ (ácidos alifáticos no identificados). Según Boom et al. (2007), la especie contiene ácido dehidroconstipático (mayor), ácido protodehidroconstipático (menor) y ácido constipático (menor).

Material examinado: Los Pirpintos, IV.1985, JJP s.n. (BAFC 39334); Copo, corticícola s/ramas jóvenes, 20.II. 1985, JJP s.n. (BAFC 39335), en bv, IX.1985, JJP s.n. (BAFC 39282), en bv, X.1985, JJP s.n. (BAFC 39283, 39284).

Obs.: Bastante frecuente en el área de estudio. Hale (1965) la ha citado para México, Guatemala, Bolivia, Brasil, Paraguay y Uruguay. Según Fleig (1997) se encuentra en Brasil en los estados de Mato Grosso do Sul, Parana y Rio Grande do Sul.

Es una especie con distribución Americana en áreas subtropicales. Osorio (1981) la citó para la Argentina (Misiones) y luego Popoff \& Ferraro (2002) la citaron para Corrientes. Ésta es la primera mención para Santiago del Estero.

17- Parmotrema praesorediosum (Nyl.) Hale, Phytologia 28: 338. 1974.

Parmelia praesorediosa Nyl., Sert. Lich. Trop. Labuan Singapore 18. 1891.

Ilustraciones: Hale (1965) pl. 5, Fig. 19; Yoshimura (1987) pl. 16, Fig. 130; Elix (1994) p. 193, Fig. 62; Popoff \& Ferraro (2002) p. 408, Fig 7.8E; Donha (2005) p. 73, Fig. 26. 
Talo corticícola o lignícola, laxamente adherido, hasta $5,0 \mathrm{~cm}$ diám., gris a gris verdoso. Lóbulos 3,0-5,0 mm de ancho, redondeados, con márgenes suberectos sin cilias. Superficie superior emaculada hasta levemente maculada, sorediada con soralios marginales y laminares punctiformes ubicados en áreas submarginales. Médula blanca. Superficie inferior negra en el centro con escasas ricinas, castaña en una franja marginal desnuda. Apotecios no vistos. Picnidios en áreas submarginales con conidios, sublageniformes 10,0-11,0 $\times 0,5 \mu \mathrm{m}$.

Química: Corteza superior $\mathrm{K}+$ amarillo (atranorina); médula $\mathrm{K}-, \mathrm{C}-, \mathrm{KC}-$ (dos ácidos alifáticos no identificados, mayores, que según David et al. (1990) son ácidos praesorediósico y protopraesorediósico).

Material examinado: Copo, VI.1985, JJP s.n. (BAFC 39333), s/ramas jóvenes de Schinopsis lorentzii en bv, , IX.1985, JJP s.n. (BAFC 39336), s/ramas jóvenes en bv, X.1985, JJP s.n. (BAFC 39337), II.1986, JJP s.n. (BAFC 39338).

Obs.: Es una especie bastante frecuente en el área de estudio. Se la considera la contraparte sorediada de P. mesotropum. Según Hale (1965) tiene una distribución muy amplia en el mundo: América de Norte y Central, islas del Caribe, América del Sur, (en particular para la Argentina hay una cita para Tucumán), África (Guinea, Congo, sur de África), India, Indonesia (Sumatra) y Japón. Krog \& Swinscow (1981) la citaron para el este de África (Uganda, Tanzania, Kenya y Etiopía). Además, de acuerdo a Elix (1994) es común en Australia tropical en sitios bastante secos y abiertos y también la ha citado para Java y Papúa Nueva Guinea. Kurokawa \& Lai (2001) la mencionan para Taiwan en China. Como se presenta en todos los continentes, aún faltando en Europa, se la puede considerar una especie con distribución Cosmopolita (Galloway, 2008) aunque fue definida por Nash \& Elix (2002) como Pantropical y Pantemplada, lo que es prácticamente coincidente. En la Argentina fue citada también para Santiago del Estero, Dpto. Copo, Los Pirpintos, por Osorio \& Ferraro (1976), y para Buenos Aires (Adler, 1988, 1992), Córdoba (Estrabou \& Adler, 1999) y Corrientes (Popoff \& Ferraro, 2002).

18- Parmotrema recipiendum (Nyl.) Hale, Phytologia 28: 338. 1974.
Parmelia recipienda Nyl., Flora 68: 609. 1885.

Rimeliella recipienda (Nyl.) Kurok., Ann. Tsukuba Bot. Gard. 10: 7. 1991.

Canomaculina recipienda (Nyl.) Elix, Mycotaxon 65: 477. 1997.

Ilustraciones: Kurokawa (1991) p. 5, Fig. 2; Elix (1994) p. 190, Fig. 60B; Popoff \& Ferraro (2002) p. 409, Fig. 7.9B.

Talo adnato en el centro, hasta 10,0 cm diám., laxamente adnato en los lóbulos, corticícola, gris mineral a gris verdoso, sin propágulos vegetativos. Lóbulos subirregulares a elongados, con ápices subredondeados, crenados, con cilias mayormente simples, infrecuentemente escuarrosas, hasta $1,5 \mathrm{~mm}$ de largo. Superficie superior con máculas efiguradas. Médula blanca. Superficie inferior castaño muy claro a beige, escasa, moderada o densamente ricinada, con ricinas dimórficas, mayormente simples y oscuras (gruesas esparcidas y largas en el centro, delgadas muy cortas hasta largas, cubriendo casi toda la superficie). Apotecios frecuentes, estipitados, disco castaño, 5,0-15,0 mm diám., con perforación central y margen liso. Ascosporas 8,0-10,0 $\times 11,0-15,0 \mu \mathrm{m}$. Picnidios frecuentes; conidios filiformes $(9,0) \quad 11,0$ $(15,0) \times 0,5 \mu \mathrm{m}$.

Química: Corteza superior $\mathrm{K}+$ amarillo (atranorina, menor a submayor); médula $\mathrm{K}-$, $\mathrm{C}-, \mathrm{KC}+$ rojo violáceo (norlobaridona, mayor, loxodina, menor).

Material examinado: Los Pirpintos, s/ Aspidosperma quebracho-blanco, 20.II.1985, JJP s.n. (BAFC 39265), IV.1985, JJP s.n. (BAFC 39318), VI.1985, JJP s.n. (BAFC 39249); Copo, corticícola, 20.II.1985, JJP s.n. (BAFC 39238), II.1985, s/Schinopsis lorentzii en bv, JJP s.n. (BAFC 39339), en bv, VI.1985, JJP s.n. (BAFC 39246, 39266, 39275).

Obs.: Parmotrema recipiendum es muy común en el área de estudio. Fue considerada una cepa química con ácido criptoclorofeico (en realidad norlobaridona) de Parmelia subcaperata por Hale (1965) quien informó su presencia en la Argentina (Jujuy, Salta, Formosa), Brasil y Paraguay. Fue citada también para Perú y Australia (Kurokawa, 1991) y confirmada para este último continente por Elix (1994), por lo que es una especie Sudamericanaaustralásica (Adler \& Calvelo, 2002). Fue citada para Misiones (Osorio, 1981) y Corrientes (Popoff 


\section{T. Adler - Líquenes Parmelioides del Parque Nacional Copo}

\& Ferraro, 2002). Ésta es la primera mención para Santiago del Estero.

19-Parmotrema reticulatum (Taylor) Choisy, Bull. Mens. Soc. Linn. Lyon 21: 175. 1952.

Parmelia reticulata Taylor, Flora Hibernica 2: 148. 1836.

Lista de sinónimos en Hale \& Fletcher, 1990. Ilustraciones: Adler (1988) p. 253, Fig. 31; Elix (1994) p. 194, Fig. 66; Donha (2005) p. 105, Fig. 40; Benatti \& Marcelli (2008) p. 80, Fig. 5a y b, y como $P$. clavuliferum p. 78 , Fig. $2 \mathrm{a}$ y b y $3 \mathrm{a}$ $\mathrm{yb}$.

Talo corticícola o lignícola, adnato a laxamente adnato, 4,0-10,0 cm diám., gris a gris verdoso. Lóbulos subirregulares, 3,0-10,0 $\mathrm{mm}$ de ancho, crenados a incisos, generalmente escasa a moderadamente ciliados, cilias cortas, $0,1-0,5 \mathrm{~mm}$ de largo, a veces hay lóbulos eciliados. Superficie superior con máculas reticulares blancas o puntos verdes, con soralios capitiformes en los márgenes, generalmente sobre lacinias cortas. Médula blanca. Superficie inferior negra, moderada a escasamente ricinada, ricinas negras, $0,1-1,0 \mathrm{~mm}$ de largo, mayormente simples y ramificadas de manera irregular, ocasionalmente escuarrosas o bifurcadas, densas en el centro, generalmente esparcidas a ausentes en una franja marginal parda a castaña debajo de los lóbulos, marfilina o variegada debajo de los lóbulos sorediados. Apotecios y picnidios no vistos.

Química: Corteza superior $\mathrm{K}+$ amarillo (atranorina); médula $\mathrm{K}+$ amarillo tornándose rojo oscuro, $\mathrm{C}-$, $\mathrm{KC}-$ (ácidos salacínico, mayor, consalacínico, menor a trazas).

Material examinado: Los Pirpintos, corticícola, IV.1985, JJP s.n. (BAFC 39342), s/Prosopis nigra, 27.V.1985, JJP s.n. (BAFC 39340), s/Aspidosperma quebracho-blanco 27.V.1985, JJP s.n. (BAFC 39341, 39359), en bv, X.1985, JJP s.n. (BAFC 39360); Copo, en bv, VI.1985, JJP s.n (BAFC 39343), en bv, X.1985, JJP s.n.(BAFC 39344).

Obs.: Los especímenes estudiados en este trabajo tienen la superficie inferior debajo de los lóbulos frecuentemente de color blanco a marfilino especialmente cuando están sorediados, que es la característica principal utilizada para diferenciar a $P$. clavuliferum (Räsänen) Streimann de $P$. reticulatum considerados sinónimos por Hale \& Fletcher (1990), lo que fue confirmado en un estudio molecular (Divakar et al., 2005). Es una especie con distribución Cosmopolita (Galloway, 2008) coincidente con la definición de Pantemplada y Pansubtropical (Hale \& Fletcher, 1990) siendo uno de los líquenes más comunes del mundo, y también en la Argentina. Fue citada para Santiago del Estero, Los Pirpintos, Dpto. Copo, por Osorio \& Ferraro (1976).

20- Parmotrema subcaperatum (Kremp.) Hale, Phytologia 28: 339. 1974.

Parmelia subcaperata Kremp., Vidensk. Meddel. Dansk Naturhist. Foren.

Kjøbenhavn 25: 10. 1874 (1873).

Rimeliella subcaperata (Kremp.) Kurok., Ann. Tsukuba Bot. Gard.10:7. 1991.

Canomaculina subcaperata (Kremp.) Elix, Mycotaxon 65: 477. 1997.

Ilustraciones: Hale (1965) pl. 14, Fig. 39, como Parmelia reparata, Kurokawa (1991) p. 8, Fig. 3, como Parmelia imperforata; Popoff \& Ferraro (2002) p. 409, Fig. 7.9D.

Talo adnato en el centro, hasta 10,0 cm diám., laxamente adnato en áreas marginales, corticícola, gris verdoso, sin propágalos vegetativos. Lóbulos 4,0-10,0 mm diám., subirregulares alargados con ápices subredondeados, crenados a incisos, ciliados, cilias mayormente simples, infrecuentemente escuarrosas, hasta $1,5 \mathrm{~mm}$ de largo. Superficie superior con maculación efigurada. Médula blanca. Superficie inferior negra a pardo claro, a veces con una ancha franja marginal beige, moderada a densamente ricinada, con ricinas dimórficas negras (pocas muy gruesas y largas en el centro y muchas delgadas y cortas cubriendo toda la superficie hasta el margen). Apotecios frecuentes, estipitados, con una perforación central, disco castaño, 5,0-15,0 mm diám., márgenes lisos. Ascosporas 12,0-15,0 $\times 18,0-20,0 \mu \mathrm{m}$. Picnidios frecuentes; conidios filiformes 13,0-14,5 x 0,5 $\mu \mathrm{m}$.

Química: Corteza superior $\mathrm{K}+$ amarillo (atranorina, menor); médula $\mathrm{K}+$ amarillo tornándose rojo oscuro (ácidos salacínico, mayor, consalacínico, menor).

Material examinado: Los Pirpintos, VI.1985, JJP s.n. (BAFC 39279); Copo, 20.II.1985, JJP s.n. 
(BAFC 39319), en bv, II.1985, JJP s.n. (BAFC 39247, 39277), IX.1985, JJP s.n. (BAFC 39278, 39351).

Obs.: La delimitación de esta especie se restringió a la cepa con ácido salacínico en la médula (Kurokawa, 1991). Es frecuente dentro del Parque Nacional. Distribuída por Australia y América del Sur (Argentina, Brasil, Paraguay, Uruguay) según Kurokawa (1991) por lo que sería una especie con distribución Sudamericana-Australásica. En la Argentina fue citada para Santiago del Estero (Osorio y Ferraro (1976, sin especificar la química), Misiones (Osorio, 1981, aclarando que se trata de la cepa con ácido salacínico) y Corrientes (Popoff \& Ferraro, 2002).

21- Parmotrema yodae (Kurok.) Hale, Mycotaxon 5: 436.1977.

Parmelia yodae Kurok, J. Coll. Arts Sci. Chiba Univ. 5: 97. 1967.

Ilustraciones: Kurokawa (1967) pl. I, Fig. D, como Parmelia yodae; Fleig (1997) p. 177, Fig. 49.

Talo folioso corticícola, gris claro a gris verdoso, laxamente adherido. Lóbulos 0,5-1,0 $\mathrm{mm}$ de ancho, subredondeados, ascendentes, márgenes crenados, ondulados, escasamente ciliados hasta cilias ausentes, cilias cortas, hasta $1,0 \mathrm{~mm}$ de largo. Superficie superior levemente maculada hasta emaculada, márgenes sorediados, soralios marginales a ligeramente submarginales. Médula blanca. Superficie inferior negra en el centro, moderadamente ricinada, con una ancha franja marginal desnuda, castaño claro, marfilino o variegada. Apotecios y picnidios no vistos; según Fleig (1997) los picnidios contienen conidios sublageniformes 6,0-7,0 $\mu \mathrm{m}$ de largo.

Química: Corteza superior $\mathrm{K}+$ amarillo (atranorina); médula $\mathrm{K}-, \mathrm{C}-, \mathrm{KC}+$ rojo violáceo (norlobaridona, mayor, loxodina, menor).

Material examinado: Los Pirpintos, corticícola s/ ramas jóvenes, VI.1985, JJP s.n. (BAFC 39287); s/ rama joven, IV.1985, JJP s.n. (BAFC 39345); Copo, corticícola, VIII.1985, JJP s.n. (BAFC 39245), II.1986, JJP s.n. (BAFC 39288).

Obs.: Parmotrema yodae es poco frecuente en el área de estudio habiendo sido mencionada y descripta para este Parque Nacional en un trabajo previo (Adler \& Calvelo, 2010). Fue descripta como especie nueva por Kurokawa en 1967 ( $P$. yodae) y luego Hale (1977) la reconoció como especie separada y no como cepa química de $P$. hababiana como lo hicieron Krog \& Swinscow (1981). Hasta la década de los 60 y anteriormente, el análisis químico se hacía por cromatografía en papel y microrrecristalización, por lo que la determinación de ácido criptoclorofeico por Hale (1965) no era fehaciente, correspondiendo a norlobaridona, según indicaron después los datos de Krog \& Swinscow (1981). Según Hale las cepas de $P$. hababiana serían dos, 1) con ácido criptoclorofeico y 2) con ácido protoliquesterínico, se distribuirían ampliamente por América del Norte, Central y del Sur y África. Los especímenes de la cepa 1 de Hale (1965) serían ejemplares de la ahora llamada $P$. yodae. Esta especie y los ejemplares determinados anteriormente como $P$. hababianum, necesitan revisión para establecer un patrón de distribución mundial fehaciente.

P. yodae puede ser considerada por ahora como una especie con distribución Afro-SudamericanaAsiática, presente en Nepal e India, Brasil (Fleig, 1997) y la Argentina (Adler \& Calvelo, 2010). Es poco frecuente en el área de estudio en Santiago del Estero, única provincia argentina para donde se la ha publicado hasta ahora.

22- Punctelia borrerina (Nyl.) Krog, Nord. J. Bot. 2: 291.1982.

Parmelia borrerina Nyl., Les Lichens des Environs de Paris: 36. 1896.

Ilustraciones: Adler (1988) p. 257, Fig. 38, como Punctelia microsticta; Canêz (2009) p. 731, Fig. 2; Canêz \& Marcelli (2010) p. 731, Fig. 2.

Talo gris mineral, adnato, hasta $10,0 \mathrm{~cm}$ diám., corticícola. Lóbulos 3,0-5,0 $\mathrm{mm}$ de ancho, márgenes eciliados. Superficie superior con pliegues con pseudocifelas, presentes también en los márgenes. Sin propágulos vegetativos. Médula blanca. Superficie inferior negra a pardo oscuro en el centro, con ricinas generalmente simples, a veces bifurcadas o penicilados, concoloras o más oscuras que el envés, frecuentemente castaño a marfilino en una franja marginal desnuda bajo los lóbulos. Apotecios frecuentes, abundantes, cortamente estipitados, disco castaño, hasta 5,0 mm diám., imperforado y con margen liso; anfitecio 


\section{T. Adler - Líquenes Parmelioides del Parque Nacional Copo}

densamente pseudocifelado. Ascosporas $(11,0)$ 13,0-16,0 × 18,0-20,0 (21). Picnidios frecuentes, agrupados en áreas submarginales; conidios filiformes $(14,0) 16,0-17,0(22,0) \times 0,5 \mu \mathrm{m}$.

Química: Corteza superior $\mathrm{K}+$ amarillo (atranorina) a $\mathrm{K}-$; médula $\mathrm{K}-\mathrm{C}-\mathrm{KC}-$ (ácido dihidropertusárico y un ácido alifático no identificado).

Material examinado: Los Pirpintos, s/Prosopis nigra, IV.1985, JJP s.n. (BAFC 37889); Copo, s/ rama joven de Acacia sp. en bv, 20.II.1985, JJP s.n. (BAFC 39346), s/rama en bv, VI.1985, JJP s.n. (BAFC 39361).

Obs.: Esta especie era conocida hasta el año 2005 como Punctelia microsticta, debido a la identificación mediante la clave de Krog (1982). Canêz (2005) y Canêz \& Marcelli (2010), a través de una lectotipificación propia, concluyeron que los ejemplares de Punctelia que carecen de propágulos vegetativos, que tienen superficie inferior mayormente negra, ascosporas de $20 \mu \mathrm{m}$ de largo o menos, con conidios filiformes largos y con ácidos alifáticos en la médula, deben llevar el nombre $P$. borrerina y no $P$. microsticta.

Es una especie bastante común en el área y en la Argentina, con distribución Sudamericana en áreas subtropicales y templadas. En Argentina fue citada como P. microsticta para Jujuy, Salta, Chaco, Misiones, Santiago del Estero, Mendoza, Córdoba, Corrientes, Santa Fe, La Pampa y Buenos Aires (Ferraro, 1986), confirmada para Buenos Aires (Adler, 1988, 1992); y La Rioja (Adler \& Calvelo, 2010). Según Krog (1982) la médula (de la especie llamada entonces $P$. microsticta) contenía ácidos alifáticos desconocidos, pero la identidad de uno de ellos fue elucidada por Maier et al. (1999).

23- Punctelia punctilla (Hale) Krog, Nord. J. Bot. 2: 291.1982.

Parmelia punctilla Hale, in Krog \& Swinscow, Norw. J. Bot. 24: 172. 1977.

Punctelia missourensis Wilhelm \& Ladd, Mycotaxon 44: 495. 1992.

Ilustraciones: Hale, en Krog \& Swinscow (1977), como Parmelia punctilla Hale, p. 172, Fig. 2; Adler (1988) p. 257, Fig. 39 (aspecto general de material saxícola); Adler (1997), p. 61, Fig. 2, p. 63 , Fig. 3 y p. 64 , Fig. 4 (detalles de material saxícola) y p. 66, Fig. 5 (detalles de material corticícola).

Talo gris mineral, adnato, hasta 15,0 cm diám., corticícola o saxícola. Lóbulos subirregulares, frecuentemente redondeados apicalmente, crenados a incisos, 2,0-8,0 $\mathrm{mm}$ de ancho. Superficie superior y márgenes con pseudocifelas punctiformes, al principio $0,05-0,20 \mathrm{~mm}$ diám., no marginadas, $0,5-0,8 \mathrm{~mm}$, en la madurez a menudo con un anillo blanco. Propágulos desarrollándose dentro de las pseudocifelas, polimórficas: incluyendo soredios, isidios papiliformes, isidios cilindroides, o más o menos ramificados, más o menos sorediados, filidios y lobulillos más o menos ramificados; tejido cortical pobremente desarrollado en los isidios cilindroides. Médula blanca. Superficie inferior castaño pálido hasta beige o marfilino hacia la parte central, usualmente más oscuro en los ápices de los lóbulos, ricinas concoloras, simples o irregularmente ramificadas. Apotecios cortamente estipitados, disco castaño, imperforado, anfitecio pseudocifelado, a veces con propágulos secundarios. Ascosporas (6,0) 7,0-9,0 (13) × (8) 10-14 (16) $\mu \mathrm{m}$. Picnidios bastante infrecuentes, cuando presentes agrupados en áreas submarginales de los lóbulos; conidios unciformes a cilíndricos $(4,0)$ 5,0-7,0 $(8,0) \mu \mathrm{m}$ de largo.

Química: Corteza superior $\mathrm{K}+$ amarillo (atranorina) a $\mathrm{K}-$; médula $\mathrm{K}-, \mathrm{C}+$ rosado a rojo, $\mathrm{KC}+$ rojo tornándose anaranjado (ácido lecanórico, mayor y compuestos relacionados, mayores a menores).

Material examinado: Los Pirpintos, s/Schinopsis lorentzii, II.1985, JJP s.n. (BAFC 37152, 37153, 37339, MOR); Copo, corticícola, II.1985, JJP s.n. (BAFC 37134), s/Acacia praecox, II.1986 (BAFC 37139, 37140, MOR). Lista completa de especímenes estudiados en Adler (1997).

Obs.: Es una especie coleccionada con frecuencia en el área de estudio. La variabilidad de la especie en cuanto a los propágulos vegetativos y el sustrato fue analizada y descripta por Adler (1997). Una actualización bastante reciente de su distribución geográfica mundial fue descripta en Adler \& Calvelo (2010), asignándole la categoría Americana-Africana. En Argentina fue citada para Buenos Aires, Mendoza, Santiago del Estero, Córdoba, Corrientes, Río Negro y Tucumán. 
1. Talo con márgenes ciliados (a veces las cilias son muy cortas y escasas, o están ausentes en ciertos lóbulos y lacinias)

$1^{\prime}$. Talo con márgenes totalmente eciliados

2. Cilias con base bulbosa Bulbothrix subcoronata

$2^{\prime}$. Cilias sin base bulbosa 3

3. Médula amarilla, cilias muy cortas en las axilas de los lóbulos Myelochroa lindmanii

3'. Médula blanca, cilias de largos diferentes, no restringidas a las axilas de los lóbulos 4

4. Superficie superior con notorias máculas efiguradas; ricinas dimórficas (de dos tipos, gruesas, esparcidas y largas en el centro del talo y muchas cortas delgadas en una ancha franja marginal) ......................... 5

4'. Superficie superior emaculada, o muy débilmente maculada sin patrón definido, o con máculas reticulares o puntos verdes sobre la superficie gris; ricinas no dimórficas

5. Médula $\mathrm{K}-, \mathrm{C}-, \mathrm{KC}-$ Parmotrema consors

5'. Médula $\mathrm{K}-, \mathrm{C}-, \mathrm{KC}+$ rosa violáceo o $\mathrm{K}+$ rojo oscuro 6

6. Médula $\mathrm{K}-, \mathrm{KC}+$ rojo violáceo (norlobaridona y loxodina)

6'. Médula $\mathrm{K}+$ rojo oscuro (ácido salacínico) Parmotrema subcaperatum

7. Talo sin propágulos vegetativos Parmotrema recipiendum

$7^{\prime}$.Talo sorediado Parmotrema conferendum

8. Talo sin máculas (o pocas muy débiles) en la superficie superior, médula $\mathrm{K}-$... 9

$8^{\prime}$. Talo con máculas reticulares o puntos verdes en la superficie superior gris, médula $\mathrm{K}+$ rojo .............. 12

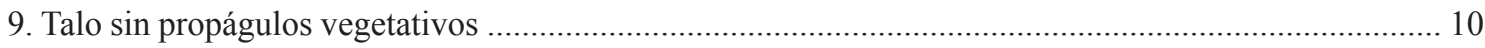

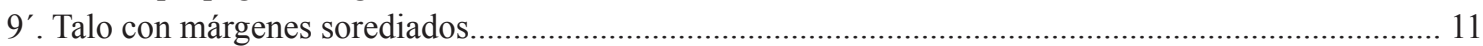

10. Lóbulos 5-15 $\mathrm{mm}$ de ancho, médula $\mathrm{UV}+$ celeste, $\mathrm{K}-, \mathrm{C}-, \mathrm{KC}+$ rojo violáceo (grupo del ácido alectorónico)

Parmotrema argentinum

10'. Lóbulos 1-2 mm ancho, médula UV-, $\mathrm{K}-, \mathrm{C}-, \mathrm{KC}+$ rosado violáceo (grupo del ácido girofórico) ......

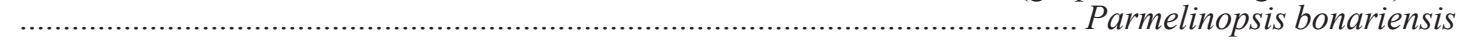

11. Médula $\mathrm{K}-, \mathrm{C}-, \mathrm{KC}-$ (ácidos alifáticos)

Parmotrema hababianum

$11^{\prime}$. Médula $\mathrm{K}-, \mathrm{C}-, \mathrm{KC}+$ rosado violáceo (norlobaridona)

Parmotrema yodae

12. Talo sorediado Parmotrema reticulatum

12'. Talo sin propágulos vegetativos 13

13. Médula con ácidos salacínico y consalacínico

Parmotrema cetratum

13'. Médula con ácidos salacínico, consalacínico, norlobaridona y loxodina ....... Parmotrema cristobaliae

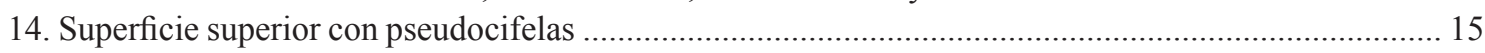

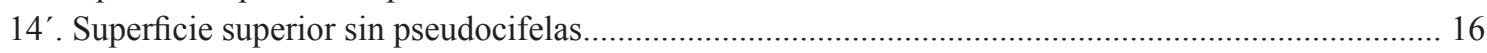

15. Talo con propágulos vegetativos polimórficos, entre lobulillos y soredios, médula $\mathrm{K}-, \mathrm{C}+$ rojo......

Punctelia punctilla

$15^{\prime}$. Talo sin propágulos vegetativos, médula $\mathrm{K}-, \mathrm{C}-$

Punctelia borrerina

16. Ricinas predominantemente dicotómicas

Hypotrachyna aspera

$16^{\prime}$. Ricinas mayormente simples, a veces mezcladas con otras no dicotómicas bifurcadas o ramificadas de manera irregular)

17. Talo sorediado

17'. Talo sin propágulos vegetativos

18. Médula $\mathrm{K}-, \mathrm{C}+$ rojo (ácido lecanórico)

Parmotrema austrosinense

$18^{\prime}$. Médula $\mathrm{K}+$ amarillo a anaranjado claro o $\mathrm{K}-, \mathrm{C}-$

19. Médula $\mathrm{K}+$ amarillo a anaranjado, grupo del ácido stíctico Canoparmelia crozalsiana

19'. Médula K-, C20

20. Médula con ácido divaricático Canoparmelia texana 


\section{T. Adler - Líquenes Parmelioides del Parque Nacional Copo}

$20^{\prime}$. Médula con ácidos alifáticos

Parmotrema praesorediosum

21. Médula $\mathrm{K}+$ rojo oscuro (con ácidos salacínico y norlobaridona) Parmotrema cristobaliae

21 '. Médula $\mathrm{K}-$ (sin ácido salacínico ni norlobaridona) 22

22. Médula $\mathrm{C}+$ rojo (ácido lecanórico) Parmotrema andinum

$22^{\prime}$. Médula $\mathrm{C}-$ 23

23. Médula con ácidos alifáticos

Parmotrema mesotropum

$23^{\prime}$. Médula con ácidos stenospórico, perlatólico y glomelliférico Canoparmelia austroamericana

\section{Conclusiones}

En este trabajo se describen por primera vez las especies parmelioides de Parmeliaceae del Parque Nacional Copo en forma conjunta. Entre ellas se cita por primera vez para la Argentina a Hypotrachyna aspera, también nueva para Santiago del Estero. Para esta provincia se mencionan por primera vez otras 12 especies: Bulbothrix subcoronata, Canoparmelia crozalsiana, C. texana, Myelochroa lindmanii, Parmotrema andinum, $P$. argentinum, $P$. conferendum, $P$. consors, $P$. cristobaliae, $P$. hababianum, $P$. mesotropum y $P$. recipiendum. Todas las especies descriptas aquí tienen como compuesto mayoritario cortical el dépsido atranorina, no habiéndose registrado ninguna especie en el área con ácido úsnico en la corteza superior, las que a veces suelen presentarse en ambientes similares en zonas más cálidas como por ejemplo en Brasil (Eliasaro, 2001). Myelochroa lindmanii es la única especie del área con médula totalmente pigmentada.

Se presenta por primera vez una clave dicotómica para diferenciar de forma sencilla las especies parmelioides del Parque Nacional Copo.

Las especies más frecuentemente coleccionadas en el Parque Nacional fueron 7 pertenecientes al género Parmotrema: $P$. andinum, $P$. austrosinense, $P$. cetratum, $P$. cristobaliae, $P$. recipiendum, $P$. reticulatum y $P$. subcaperatum.

Las categorías biogeográficas mejor representadas son las de amplia distribución en el globo terrestre (Cosmopolita, Pantropical, Pantemplada, Pansubtropical) con 6 especies: cinco sorediadas, Canoparmelia crozalsiana, C. texana, Parmotrema austrosinense, $P$. praesorediosum y $P$. reticulatum, y una sola sin propágulos vegetativos, $P$. cetratum. Siguen en orden de importancia las categorías: Endémica, del Sur de América del Sur (3), Sudamericana-Australásica (3), Sudamericana (3), Americana-Africana (3),
Americana (2), Afro-Sudamericana-Asiática (2) y Americana-Asiática (1). Los endemismos (Canoparmelia austroamericana, Parmelinopsis bonariensis y Parmotrema cristobaliae) carecen de propágulos vegetativos al igual que las especies Sudamericanas-Australásicas (Parmotrema. consors, $P$. recipiendum y P. subcaperatum).

\section{Agradecimientos}

Al CONICET (Consejo Nacional de Investigaciones Científicas y Técnicas de Argentina por el apoyo financiero (PIP 1482) y a la Facultad de Ciencias Exactas y Naturales (UBA) por el lugar de trabajo, dentro de la Unidad Ejecutora PROPLAME-PRHIDEB (CONICETUBA). M. T. Adler es miembro de la Carrera del Investigador (CONICET). También se agradecen las observaciones y sugerencias de los revisores anónimos que permitieron mejorar la versión final de este trabajo.

\section{Bibliografía}

ADLER, M. T. 1987. A new species of the genus Canoparmelia from Argentina. Mycotaxon 28: 251254.

ADLER, M. T. 1988. La familia Parmeliaceae (Líquenes, Ascomycotina) en la Provincia de Buenos Aires: estudio taxonómico-florístico. Tesis de Doctorado en Ciencias Biológicas. Facultad de Ciencias Exactas y Naturales, Universidad de Buenos Aires.

ADLER, M. T. 1989. Two new species in Parmeliaceae (Lichenized Ascomycotina) and new records for Argentina. Mycotaxon 35: 399-404.

ADLER, M. T. 1992. Clave de los géneros y las especies de Parmeliaceae (Líchenes, Ascomycotina) de la provincia de Buenos Aires (Argentina). Bol. Soc. Argent. Bot.28: 11-17.

ADLER, M. T. 1996. A comparative study on Punctelia colombiana and Punctelia stictica (Parmeliaceae, 
Lichenized Ascomycotina). Mycotaxon 58:77-92.

ADLER, M. T. 1997. Polymorphism of vegetative propagules in Punctelia punctilla (Parmeliaceae, Lecanorales) and the delimitation of the species. Mycotaxon 63: 57-70.

ADLER, M. T. \& T. AHTI. 1996. The distinction of Punctelia perreticulata and P. subrudecta (Parmeliaceae, Lecanorales). Lichenologist 28: 431436.

ADLER, M. T. \& S. CALVELO. 2002. Parmeliaceae s. str. (Lichenized Ascomycetes) from Tierra del Fuego (southern South America) and their distribution patterns. Mitt. Inst. Allg. Bot. Hamburg 30-32: 9-24.

ADLER, M. T. \& S. CALVELO. 2007. Flavoparmelia amplexa and $F$. springtonensis (Parmeliaceae) new to the Americas and additions to the lichen flora of Argentina. Bibl. Lichenol. 95: 121-129.

ADLER, M. T. \& S. CALVELO. 2010. Flavoparmelia baltimorensis, Parmotrema yodae y Xanthoparmelia braziliensis, primeras citas para Argentina y ampliación de distribuciones para otras Parmeliaceae (Ascomycota liquenizados). Bol. Soc. Argent. Bot. 45: 5-16.

ADLER, M. T. \& J. A. ELIX. 1987. Three new saxicolous species in Parmeliaceae (Lichenized Ascomycotina) from Argentina. Mycotaxon 30: 339-344.

ADLER, M. T. \& J. A. ELIX. 1992. New records of Hypotrachyna and Parmelinopsis lichens (Ascomycotina, Parmeliaceae) from North-West and central Argentina. Mycotaxon 43: 283-288.

BENATTI, M. N. 2012. A review of the genus Bulbothrix Hale: the species with medullary norstictic or protocetraric acids. MycoKeys 2: 1-28.

BENATTI, M. N. \& M. P. MARCELLI. 2008. Espécies de Parmotrema (Parmeliaceae, Ascomycetes liquenizados) com máculas reticulares do litoral centro-sul do estado São Paulo, Brasil. Hoehnea 35: 75-90.

BOOM, P. P. G. VAN DEN, J. A. ELIX \& H. J. M. SIPMAN. 2007. New or interesting lichen records from Guatemala. I. Willdenowia 37: 363-375.

BRUMMIT, R. \& C. POWELL.1992. Authors of Plant names. Royal Botanical Garden, Kew.

CABRERA, A. \& A. WILLINK. 1980. Biogeografía de América Latina, $2^{\mathrm{a}}$ ed. Monografías O.E.A. 13, Washington, DC.

CALVELO, S. \& M. T. ADLER. 1999. Parmelia araucana sp. nov. and new reports in the Parmeliaceae sensu stricto (lichenized Ascomycotina) from Patagonia and Tierra del Fuego (Argentina). Sydowia 51: 145-154.

CALVELO, S. \& S. LIBERATORE. 2002. Catálogo de los líquenes de la Argentina. Kurtziana 29: 7-170.

CANÊZ, L. 2005. A familia Parmeliaceae na localidade de fazenda de Estrela, municipio de Vacaria, Rio Grande do Sul, Brasil. Dissertaçao (mestrado) Instituto de
Botânica da Secretaria de Estado de Meio Ambiente, São Paulo.

CANÊZ, L. 2009. Estudos taxonômicos em Punctelia (Parmeliaceae, Ascomycetes Liquenizados). Tese de Doutorado em Biodiversidade Vegetal e Meio Ambiente, na Área de Concentraçao de Plantas Avasculares e Fungos em Análises Ambientais. Instituto de Botânica da Secretaria do Meio Ambiente, São Paulo.

CANÊZ, L. \& M. P. MARCELLI. 2010. The Punctelia microsticta-group (Parmeliaceae). Bryologist 113: 728-738.

CHEN, J-B., S-L., WANG \& J.A. ELIX. 2005. Parmeliaceae (Ascomycota) lichens in China's mainland III. The genus Parmotrema. Mycotaxon 91: 93-113.

CRESPO, A., F. KAUFF, P. K. DIVAKAR, R. DEL PRADO, S. PÉREZ-ORTEGA, G. AMO DE PAZ, Z. FERENCOVA, O. BLANCO, B. ROCA-VALIENTE, J. NÚÑEZ-ZAPATA, P. CUBAS, A. ARGÜELLO, J. A. ELIX, T. L. ESSLINGER, D. L. HAWKSWORTH, A. MILLANES, M.C. MOLINA, M. WEDIN, T. AHTI, A.APTROOT, E. BARRENO, F. BUNGARTZ, S. CALVELO, M. CANDAN, M. COLE, D. ERTZ, B. GOFFINET, L. LINDLOM, R. LÜCKING, F. LUTZONI, J-E. MATTSON, M. I. MESSUTTI, J. MIADLIKOWSKA, M. PIERCEY-NORMORE, V. J. RICO, H. J. M. SIPMAN, I. SCHMITT, T. SPRIBILLE, A. THELL, G. THOR, D. K. UPRETI \& H. T. LUMBSCH. 2010. Phylogenetic generic classification of parmelioid lichens (Parmeliaceae, Ascomycota) based on molecular, morphological and chemical evidence. Taxon 59: 1735-1753.

CULBERSON, C.F. \& K. AMMANN. 1979. Standardmethode zur Dünnschichtchromatographie von Flechtensubstanzen. Herzogia 5: 273-314.

DAVID, F., J. A. ELIX \& M. W. BINSAMSUDIN. 1990. Two new aliphatic acids from the lichen Parmotrema praesorediosum. Aust. J. Chem. 43: 1297-1300.

DIVAKAR, P. K., O. BLANCO, D. L. HAWKSWORTH \& A. CRESPO. 2005. Molecular phylogenetic studies on the Parmotrema reticulatum (syn. Rimelia reticulata) complex, including the confirmation of $P$. pseudoreticulatum as a distinct species. Lichenologist 37: 55-65.

DONHA, C. G. 2005. Os géneros Canomaculina, Parmotrema e Rimelia (Ascomycota liquenizados, Parmeliaceae) na área de proteção ambiaental de Gauaraqueçaba-Parana-Brasil. Dissertação para ou Mestrado. UFPR, Curitiba.

ELIASARO, S. 2001. Estudio taxonómico y florístico sobre las Parmeliaceae sensu stricto (Ascomycota liquenizados) del Segundo Planalto del estado de Paraná, Brasil. Tesis de Doctorado en Ciencias Biológicas. Facultad de Ciencias Exactas y Naturales, Universidad de Buenos Aires. 


\section{T. Adler - Líquenes Parmelioides del Parque Nacional Copo}

ELIASARO, S. \& C. G. DONHA. 2003. The genera Canomaculina and Parmotrema (Parmeliaceae, Lichenized Ascomycota) in Curitiba, Paraná State, Brazil. Revista Brasil. Bot. 26: 239-247.

ELIASARO, S., L. M. CRUZ, M. IACOMIMI, F. OLIVEIRA PEDROSA \& L. M. C. CORDEIRO. 2010. Phylogenetic relationship of Parmelia lindmanii (Parmeliaceae) inferred by analysis of its nuITS rDNA sequence. Lichenologist 42: 423-428.

ELIX, J. A. 1994. Flora of Australia 55, Lichens-Lecanorales 2, Parmeliaceae. Australian National Resources Study, Canberra.

ELIX, J. A. 1997. The lichen genera Canomaculina and Rimeliella (Ascomycotina, Parmeliaceae). Mycotaxon 65: 475-479.

ELIX, J. A. \& M. T. ADLER. 1987. A new species of Flavoparmelia and Flavopunctelia (Lichenized Ascomycota) from Argentina. Mycotaxon 30: 335338.

ELIX, J. A. \& M. E. HALE. 1987. Canomaculina, Myelochroa, Parmelinella, Parmelinopsis and Parmotremopsis, five new genera in the Parmeliaceae (lichenized Ascomycotina). Mycotaxon 29: 233-244.

ELIX, J. A., E. FISCHER \& D. KILLMAN. 2005. New saxicolous species of Hypotrachyna and Parmotrema (Parmeliaceae) from Rwanda. Lichenologist 37: 101-104.

ESTRABOU, C \& M. T. ADLER. 1999. Novedades sobre Parmeliaceae sensu stricto (Ascomycota Liquenizados) de la provincial de Córdoba, República Argentina. Bol. Soc. Argent. Bot. 34: 63-73.

ESTRABOU, C. \& M. T. ADLER. 2000. Two new species of Parmotrema (Parmeliaceae, Lichenized Ascomycotina) from Argentina. Mycotaxon 66: 131136.

ESTRABOU, C., L. STIEFKENS, M. HADID, J. M. RODRÍGUEZ \& A. PÉREZ. 2005. Estudio comparativo de la comunidad liquénica en cuatro ecosistemas de la provincia de Córdoba. Bol. Soc. Argent. Bot. 40: 3-12.

FERRARO, L. I. 1979. Una nueva especie de Parmeliaceae para el NE argentino. Hickenia 1: 191-193.

FERRARO, L. I. 1981. Contribución al estudio de las Parmeliaceas (Líquenes) de Corrientes, Rep. Argentina. Bonplandia 5: 83-99.

FERRARO, L. I. 1986. Contribution to the study of Argentine Parmeliaceae. The genus Punctelia Krog and Flavopunctelia (Krog) Hale. Phytologia 61: 189203.

FERRARO, L. I. \& J. A. ELIX. 1993. Two new species of Parmeliaceae (Lichenized Ascomycotina) from South America. Mycotaxon 49: 405-409.

FERRARO, L. I. \& J. A. ELIX. 2000. A new species of Canomaculina (lichenized, Ascomycotina,
Parmeliaceae) from Argentina. Mycotaxon 74: 391394.

FLEIG, M. 1997. Os gêneros Parmotrema, Rimelia e Rimeliella (Lichenes-Ascomycotina, Parmeliaceae) no Rio Grande do Sul, Brasil. Tese de Doutorado. Instituto de Biociências da Universidade de São Paulo.

GALLOWAY. D. J. 2008. Lichen Biogeography. In: T. H. Nash III (ed.): Lichen Biology, pp.199-216. Cambridge University Press, Cambridge.

HALE, M. E. 1965. A monograph of Parmelia subgenus Amphigymnia. Contrib. US Natnl. Herb. 36: 193-358.

HALE, M. E. 1976a. A monograph of the lichen genus Bulbothrix Hale (Parmeliaceae). Smithsonian Contr. Bot. 32: 1-29.

HALE, M. E. 1976b. A monograph of the lichen genus Pseudoparmelia Lynge (Parmeliaceae). Smithsonian Contr. Bot. 31: 1-61.

HALE, M. E. 1976c. A monograph of the genus Parmelina Hale (Parmeliaceae). Smithsonian Contr. Bot. 33: $1-60$.

HALE, M. E. 1977. New species in the lichen genus Parmotrema Mass. Mycotaxon 5: 432-448.

HALE, M. E. \& A. FLECTCHER.1990. Rimelia Hale \& Fletcher, a new lichen genus (Ascomycotina: Parmeliaceae). Bryologist 93: 23-29.

HOLMGREN, P. K. \& N. H. HOLMGREN. 2001. Index Herbariorum. Part I: The herbaria of the World, ed. 8. Regnum Veg. 120. New York Botanical Garden, New York.

JUNGBLUTH, P., M. P. MARCELLI \& J. A. ELIX. 2008. Five new species of Bulbothrix (Parmeliaceae) from cerrado vegetation in São Paulo State, Brazil. Mycotaxon 104: 51-63.

KROG, H. 1982. Punctelia, a new lichen genus in the Parmeliaceae. Nord. J. Bot. 2: 287-292.

KROG, H. \& T. D. V. SWINSCOW. 1977. The Parmelia borreri group in East Africa. Norweg. J. Bot. 24: 167-177.

KROG, H. \& T.D.V. SWINSCOW. 1981. Parmelia subgenus Amphigymnia (lichens) in East Africa. Bull. Brit. Mus. (Nat. Hist.), Bot. ser., 9: 143-231.

KUKWA, M., K. BACH, J. M. SIPMAN \& A. FLAKUS. 2012. Thirty-six species of the lichen genus Parmotrema (Lecanorales, Ascomycota) new to Bolivia. Polish Bot. J. 57: 243-257.

KUROKAWA, S. 1967. Foliose lichens collected by Dr. K. Yoda in the Rolwaling Himal. Nepal. J. Coll. Arts Sci. Chiba Univ. Nat. Sci. 5: 93-97.

KUROKAWA, S. 1991. Rimeliella, a new lichen genus related to Rimelia of the Parmeliaceae. Ann. Tsukuba Bot. Gard. 10: 1-14.

KUROKAWA, S. \& M-J. LAI. 2001. Parmelioid lichen genera and species in Taiwan. Mycotaxon 77: 225-284.

LENDEMER, C. J. \& B. P. HODKINSON. 2012. Recognition of the Parmelia crozalsiana group as the 
genus Crespoa. North Amer. Fungi 7: 1-5.

LÓPEZ DE CASENAVE, J., J. P. PELOTTO \& J. J. PROTOMASTRO. 1995. Edge-interior differences in vegetation structure and composition in a Chaco semiarid forest, Argentina. Forest Ecol. Manag. 72: 61-69.

LOUWHOFF, S. H. J. J. 2001. Biogeography of Hypotrachyna, Parmotrema and allied genera (Parmeliaceae) in the Pacific Islands. Bibl. Lichenol. 78: 223-246.

MAIER, M. S., D. I. GONZÁLEZ MARIMÓN, C. A. STORTZ \& M. T. ADLER. 1999. A revised structure for (-)-Dihydropertusaric acid, a $\gamma$-butyrolactone acid from the lichen Punctelia microsticta. J. Nat. Prod. 62: 1565-1567.

MARCELLI, M. P. \& C. H. RIBEIRO.2002. Twenty-one new species of Parmeliaceae (lichenized fungi) from Southeastern Brazil. Mitt. Inst. Allg. Bot. Hamburg 30-32: 125-155.

MICHLIG, A. \& L. I. FERRARO. 2010. The first record of Parmotrema pseudocrinitum (Parmeliaceae, lichenized Ascomycotina) in South America. Mycotaxon 112: 275-282.

MICHLIG, A. \& L. I. FERRARO. 2012a. Diversidad de macrolíquenes del Parque Nacional Mburucuyá (Corrientes, Argentina). Bol. Soc. Argent. Bot. 47: 287-302.

MICHLIG, A. \& L. I. FERRARO. 2012b. Nuevos registros de especies sorediadas de Parmotrema con ácido protocetrárico (Ascomycota, Parmeliaceae) en el Sur de Sudamérica. Darwiniana 50: 323-331.

NASH, T. H. \& J. A. ELIX. 2002. Parmotrema. In: NASH III, T. H., B. D. RYAN, C. GRIES, F. BUNGARTZ (eds.), Lichen Flora of the Greater Sonoran Desert Region, Vol I, pp. 318-329. Arizona State University, Tempe.

OSORIO, H. 1969. Contribution to the lichen flora of Argentina-III. Additions. Bryologist 72: 409-410.

OSORIO, H. 1970. Contribution to the lichen flora of Argentina V. Some new records. Com. bot. Mus. hist. nat. Montev. IV (54): 1-2.

OSORIO, H. 1975. Contribution to the lichen flora of Argentina. VI. Lichens from Concordia, Entre Ríos Province. Rev. Bryol. et Lichenol. 41: 83- 85.

OSORIO, H. 1981. Contribution to the lichen flora of Argentina XIII. Lichen from Misiones Province. Com. bot. Mus. Nat .hist. Montev. 64: 1-18.

OSORIO, H. \& F. R. DESPÓSITO. 1979. Contribution to the lichen flora of Argentina. 11: Lichens from Cabo San Antonio, Buenos Aires Province. Mycotaxon 8: 239-242.

OSORIO, H. \& L. I. FERRARO. 1975. Contribution to the lichen flora of Argentina VII. New and noteworthy records from the Province of Corrientes. Com. bot. Museo hist. nat. Montev. IV (57): 1-4.

OSORIO, H. \& L. I. FERRARO. 1976. Contribution to the lichen flora of Argentina. IX. Some lichens from the Provinces of Santa Fe and Santiago del Estero. Mycotaxon 4: 331-334.

OSORIO, H. \& L. I. FERRARO. 2001. Contribution to the lichen flora of Argentina. XX. Lichens from the Province of Jujuy. Com. bot. Mus. nac. hist. nat. antrop. VI (118): 1-7.

POPOFF, O. \& L. I. FERRARO. 2002. Hongos y Líquenes. En: Arbo, M. M. \& S. G. Tressens (eds.), Flora del Iberá, pp. 381-415. EUDENE, Buenos Aires.

PRADO, I. A. 1993. What is the Gran Chaco vegetation in South America. (review). Contribution to the study of flora and vegetation of the Chaco. V. Candollea 48: 145-172.

PROTOMASTRO, J. J. 1988. Fenología y mecanismos de interacción en el bosque de quebracho colorado, blanco y mistol. Tesis de Doctorado en Ciencias Biológicas. Facultad de Ciencias Exactas y Naturales, Universidad de Buenos Aires.

QUILHOT, W., I. PEREIRA, G. GUZMÁN, R. RODRÍGUEZ \& I. SEREY. 1998. Categorías de conservación de líquenes nativos de Chile (Reunión de trabajo con especialistas nacionales agosto 1998). Bol. Mus. Nac. Hist. Nat. 47: 9-22.

SIPMAN, H. J., J. A. \& T. H. NASH III. 2009. Hypotrachyna (Parmeliaceae, Lichenized Fungi). Flora Neotropica Monographs 104. New York Botanical Garden, New York.

SPIELMANN, A. A. 2009. Estudos taxonômicos em Parmotrema s. 1. (Parmeliaceae, Ascomycota liquenizados) com ácido salazínico. Tese de Doutorado em Biodiversidade Vegetal e Meio Ambiente, na Área de Concentraçao de Plantas Avasculares e Fungos. Instituto de Botânica da Secretaría do Meio Ambiente, São Paulo.

SWINSCOW, T. D. V. \& H. KROG. 1988. Macrolichens of East Africa. British Museum (Natural History), London.

TEHLER, A. \& M. WEDIN. 2008. Systematics of lichenized fungi, in Lichen Biology (T. H. Nash ed.) pp 336-352. Cambridge Univ. Press, New York.

THELL, A., B. HERBER, A. APTROOT, M. T. ADLER, T. FEUERER \& E. I. KÄRNEFELT. 2005. A preliminary phylogeographic study of Flavopunctelia and Punctelia inferred from rDNA ITS-sequences. Folia Cryptog. Estonica 41: 115-122.

YOSHIMURA, I. 1987. Lichen Flora of Japan in color. Hoikusha Publ., Osaka.

Recibido el 4 de diciembre de 2012, aceptado el 14 de mayo de 2013. 